\title{
Characterizations of Sets of Finite Perimeter Using Heat Kernels in Metric Spaces
}

\section{Marola, Niko}

2016-11

Marola , N , Miranda , M \& Shanmugalingam , N 2016, ' Characterizations of Sets of Finite Perimeter Using Heat Kernels in Metric Spaces ' , Potential Analysis, vol. 45 , no. 4 , pp. 609-633 . https://doi.org/10.1007/s11118-016-9560-3

http://hdl.handle.net/10138/224137

https://doi.org/10.1007/s11118-016-9560-3

other

acceptedVersion

Downloaded from Helda, University of Helsinki institutional repository.

This is an electronic reprint of the original article.

This reprint may differ from the original in pagination and typographic detail.

Please cite the original version. 


\title{
CHARACTERIZATIONS OF SETS OF FINITE PERIMETER USING HEAT KERNELS IN METRIC SPACES
}

\author{
NIKO MAROLA, MICHELE MIRANDA JR., AND NAGESWARI SHANMUGALINGAM
}

\begin{abstract}
The overarching goal of this paper is to link the notion of sets of finite perimeter (a concept associated with $N^{1,1}$-spaces) and the theory of heat semigroups (a concept related to $N^{1,2}$-spaces) in the setting of metric measure spaces whose measure is doubling and supports a 1-Poincaré inequality. We prove a characterization of sets of finite perimeter in terms of a short time behavior of the heat semigroup in such metric spaces. We also give a new characterization of BV functions in terms of a near-diagonal energy in this general setting.
\end{abstract}

\section{CONTENTS}

1. Introduction

1.1. Ledoux-type characterization

1.2. De Giorgi-type characterization

1.3. Organization of the paper

2. Basic concepts

2.1. Standing assumptions

2.2. Differentiable structure

2.3. Semigroup associated with $\mathcal{E}$

2.4. $\mathrm{BV}$ functions and sets of finite perimeter 8

3. A characterization of BV functions 9

4. Sets of finite perimeter: A Ledoux type characterization 14

5. Total variation: De Giorgi characterization under Bakry-Émery condition 19

6. Appendix 21

References 26

\section{INTRODUCTION}

In this paper we study functions of bounded variation and, in particular, sets of finite perimeter on general metric measure spaces. More precisely, we investigate a relation between the perimeter of a set and the short-time behavior of the action of a heat

2010 Mathematics Subject Classification. 31C25, 26B30, 46E35, 35K05.

Key words and phrases. Bakry-Émery condition, bounded variation, Dirichlet form, doubling measure, heat kernel, heat semigroup, isoperimetric inequality, metric space, perimeter, Poincaré inequality, sets of finite perimeter, total variation. 
semigroup on the characteristic function of such a set. First, we provide in Theorem 3.1 a new characterization of BV functions in terms of asymptotic behavior of a near-diagonal energy or, in other words, the near-diagonal part of the Korevaar-Schoen type energy [KS]: Suppose that $u \in L^{1}(X)$, then $u \in \mathrm{BV}(X)$ if, and only if,

$$
\liminf _{\varepsilon \rightarrow 0^{+}} \frac{1}{\varepsilon} \int_{\Delta^{\varepsilon}} \frac{|u(x)-u(y)|}{\sqrt{\mu\left(B_{\varepsilon}(x)\right)} \sqrt{\mu\left(B_{\varepsilon}(y)\right)}} d \mu(x, y)<\infty .
$$

Here $\Delta^{\varepsilon}, \varepsilon>0$, denotes the $\varepsilon$-neighborhood of the diagonal in $X \times X$.

Second, we shall give in Theorem 4.1 a Ledoux-type characterization of sets of finite perimeter in terms of the heat semigroup [L], with the focus again on the near-diagonal part of the boundary of the set. To motivate the discussion below let us state at this point the main parts of Theorem 4.1: If

$$
\liminf _{t \rightarrow 0^{+}} \frac{1}{\sqrt{t}} \int_{E \sqrt{t} \backslash E} T_{t} \chi_{E}(x) d \mu(x)<\infty,
$$

then $E$ is of finite perimeter. On the other hand, if $E$ is a set of finite perimeter and satisfies an extra assumution in (4.2), then $E$ must satisfy the inequality above.

The characterizations obtained in this paper connect the theory of functions of bounded variation and sets of finite perimeter to the theory of the heat semigroup and heat flow in metric spaces, thus connecting the nonlinear potential theory associated with the index $p=1$ to the linear potential theory associated with the index $p=2$. In the Euclidean setting such a connection is well-established, see for example [DeG] and [L]. In the setting of Riemannian manifolds with lower bounded Ricci curvature, see [CM], [MP], and [MP2]. In the more general metric setting there is precedence; indeed, it was shown in $[\mathrm{JK}]$ that when the measure on a metric measure space $X$ is doubling, supports a 2Poincaré inequality, and satisfies a curvature condition, a global isoperimetric inequality holds in $X$. In this note we are more interested in characterizing sets of finite perimeter in terms of the asymptotic behavior of the heat extension of the characteristic function of the sets, and to do so we need the stronger assumption of 1-Poincaré inequality. However, we avoid the use of curvature conditions by focusing on near-diagonal part of the set in question. It is not known whether the curvature condition assumed in [JK], together with the doubling property of the measure and the support of a 2-Poincaré inequality, implies the support of a 1-Poincaré inequality.

1.1. Ledoux-type characterization. It was observed by Ledoux in [L] that the classical isoperimetric inequality in $\mathbb{R}^{n}$, and also in more general Gauss spaces, can be characterized by the fact that the $L^{2}$-norm of the heat semigroup acting on the characteristic function of sets is increasing under isoperimetric rearrangement. More precisely, condition that a set $B$ is isoperimetric, that is a minimizer of the perimeter measure among all sets $E$ with the same measure as $B$, is equivalent to the following $L^{2}$-inequality

$$
\left\|T_{t} \chi_{E}\right\|_{L^{2}\left(\mathbb{R}^{n}\right)} \leq\left\|T_{t} \chi_{B}\right\|_{L^{2}\left(\mathbb{R}^{n}\right)}
$$

for all $t \geq 0$ and sets $E \subset \mathbb{R}^{n}$ with the same volume as the Euclidean ball $B$. Here $\chi_{E}$ denotes the characteristic function of the set $E$, and $T_{t}$ stands for the heat semigroup 
defined on $L^{2}\left(\mathbb{R}^{n}\right)$ by convolution with the classical Gauss-Weierstrass kernel so that $T_{t} f$ solves the Cauchy problem

$$
\left\{\begin{array}{l}
\partial_{t} u=\Delta u \\
u(0, x)=f(x)
\end{array}\right.
$$

with $f \in L^{2}\left(\mathbb{R}^{n}\right)$. In particular, it was shown in $[\mathrm{L}]$ (the reader is recommended to also see the paper $[\mathrm{P}]$ by Preunkert) that

$$
\lim _{t \rightarrow 0^{+}} \sqrt{\frac{\pi}{t}} \int_{\mathbb{R}^{n} \backslash B} T_{t} \chi_{B}(x) d x=P(B),
$$

whenever $B$ is a Euclidean ball and $P(B)$ the perimeter of $B$ in $\mathbb{R}^{n}$. Moreover, the inequality

$$
\sqrt{\frac{\pi}{t}} \int_{\mathbb{R}^{n} \backslash E} T_{t} \chi_{E}(x) d x \leq P(E)
$$

holds for every $t \geq 0$ and for all subsets $E$ of $\mathbb{R}^{n}$ with finite measure.

The authors in [MP2] pursued the investigation of the relation between the perimeter of a set and the short-time behavior of the heat semigroup as described in (1.1). They observed, by considering the measure-theoretic properties of the reduced boundary of a set, that equality (1.1) is actually valid for all sets of finite perimeter. In addition, the finiteness of the limit on the left hand side in (1.1) is enough to characterize sets of finite perimeter in $\mathbb{R}^{n}$. Similar characterizations have also been obtained in $[\mathrm{B}],[\mathrm{D}]$, and $[\mathrm{P}-\mathrm{P}]$, where more general convolution kernels than the classical Gauss-Weierstrass kernel were considered. On the other hand, the approach taken in [MP2] has more geometric flavor.

In the present paper, we study characterizations of sets of finite perimeter and hence, by the co-area formula, all functions in the BV-class, in terms of the heat semigroup. The recent results in $[\mathrm{JK}]$ demonstrate that under some curvature assumptions on the metric measure space, if the space is also doubling in measure and supports a 2-Poincaré inequality, a (global) isoperimetric inequality follows. Adding this to the discussion above, it is reasonable to ask whether the notion of a set of finite perimeter in a metric measure space (first defined in $[\mathrm{Mr}]$ ), using $L^{1}$-approximations by Lipschitz functions, is connected to the behavior of heat extension of the characteristic function of the set. In this paper we show that a Ledoux-type characterization of sets of finite perimeter in terms of the heat extension of the characteristic function of such sets is possible if the measure on the space is doubling and supports a 1-Poincaré inequality.

The Ledoux characterization in the limit of (1.1) requires global information of the decay of the heat extension. So if the space is hyperbolic then the behavior of $E$ and $\mathbb{R}^{n} \backslash E$ far away from $\partial E$ also might play a role in the limit given in (1.1). We therefore modify the criterion and consider only regions near $\partial E$ as in (4.1).

1.2. De Giorgi-type characterization. In the Euclidean setting the original definition of sets of finite perimeter in terms of heat extension, is due to De Giorgi [DeG]. In Section 5 we consider a characterization of total variation and BV functions. Such a characterization is related to the definition of sets of finite perimeter considered by 
De Giorgi [DeG] making use of a regularization procedure based on the heat kernel. Indeed, for a function $u \in L^{1}\left(\mathbb{R}^{n}\right)$ the limit

$$
\lim _{t \rightarrow 0^{+}} \int_{\mathbb{R}^{n}}\left|\nabla T_{t} u(x)\right| d x
$$

exists and is finite if, and only if, the distributional gradient of $u$ is an $\mathbb{R}^{n}$-valued measure $D u$ with finite total variation $|D u|\left(\mathbb{R}^{n}\right)$. Moreover, the limit in (1.2) equals $|D u|\left(\mathbb{R}^{n}\right)$. This result has been generalized to the setting of Riemannian manifolds in $[\mathrm{CM}]$, with the restriction that the Ricci curvature of a manifold is bounded from below. We refer also to the result in $[\mathrm{MP}]$, where further bounds on the geometry of the manifold were assumed. We refer also to a recent paper [GP] where a more general condition on the Ricci curvature has been considered, that is the Ricci tensor can be splitted into a sum of two terms, one which is bounded from below and the second one belonging to a suitable Kato class. We mention, in passing, that in the setting of Carnot groups the authors of $[\mathrm{BMP}]$ showed that the aforementioned result is valid in a weaker sense, namely that both the limit inferior and superior are comparable to the total variation of $u$, but it is not known whether equality holds.

In Section 5, we shall give a generalization of the preceding result to metric measure spaces as discussed above by imposing an additional assumption on a Dirichlet form $\mathcal{E}$ compatible with the Cheeger differentiable structure and associated with $X$. We shall assume that the Dirichlet form as considered above satisfies the Bakry-Émery condition $\operatorname{BE}(K, \infty)$ (see Definition 5.1), for some $K \in \mathbb{R}$. Providing the analogous selfimprovement property of the $\mathrm{BE}(K, \infty)$ condition as obtained by Savaré in a recent paper [S], we obtain in Proposition 5.2 a metric space version of the De Giorgi characterization of the total variation of a BV function. We point out here, however, that the condition $\mathrm{BE}(K, \infty)$ is not satisfied by some Carnot groups, for example Heisenberg groups, and so our discussion does not overlap with that of $[\mathrm{BMP}]$. Recall that a 1-Poincaré inequality follows from the $\operatorname{BE}(K, \infty)$ condition, or even from weaker curvature conditions like the $\mathrm{CD}(K, \infty)$ condition of Lott-Sturm-Villani. However, a complete separable metric space endowed with a probability measure and satisfying the $\mathrm{BE}(K, \infty)$ condition need not be doubling.

1.3. Organization of the paper. We have organized our paper as follows. In Section 2 we recall the tools needed for our analysis in metric measure spaces as well as the basic properties of the heat semigroup and BV functions in this setting. Our main results are then stated in Theorem 3.1 and Theorem 4.1 in Section 3 and Section 4, respectively. In Section 5 we consider a characterization of total variation and BV functions. In the appendix, see Section 6, we gather together properties of the Bakry-Émery condition needed in Section 5.

Acknowledgement. The research was mostly carried out during the stay of the three authors at Institut Mittag-Leffler, Sweden, in Fall 2013, and finalized while N.M. and M.M. were visiting N.S. at the University of Cincinnati, USA, in Spring 2014. The authors wish to thank these both institutions for the support and kind hospitality. 
N.M. was supported by the Academy of Finland and the Väisälä Foundation. The research of M.M. was supported partially by the PRIN 2012 and partially by FAR grant of the Department of Mathematics and Computer Science of University of Ferrara. N.S. was partially funded by NSF grant \#DMS-1200915.

\section{BASIC CONCEPTS}

In this section we recall the basic concepts that allow for nonsmooth analysis on metric measure spaces.

2.1. Standing assumptions. Throughout the paper we will assume that $(X, d, \mu)$ is a complete metric measure space equipped with a Borel regular doubling measure $\mu$ supporting a 1-Poincaré inequality.

Recall that a Borel-regular measure $\mu$ is doubling if there exists a constant $c_{D} \geq 1$ such that for every ball $B_{r}(x):=B(x, r)=\{y \in X: d(x, y)<r\}, x \in X$ and $r>0$,

$$
0<\mu\left(B_{2 r}(x)\right) \leq c_{D} \mu\left(B_{r}(x)\right)<\infty .
$$

Moreover, $(X, d, \mu)$ supports a 1-Poincaré inequality if there exist constants $c>0$ and $\lambda \geq 1$ such that for any $u \in \operatorname{Lip}(X)$ (real-valued Lipschitz continuous function on $X$ ), the inequality

$$
\int_{B_{r}(x)}\left|u(y)-u_{B_{r}(x)}\right| d \mu(y) \leq c_{P} r \int_{B_{\lambda r}(x)} \operatorname{lip}(u)(y) d \mu(y)
$$

holds, where $\operatorname{lip}(u)$ is the local Lipschitz constant of $u$ defined as

$$
\operatorname{lip}(u)(y):=\liminf _{\varrho \rightarrow 0^{+}} \sup _{z \in B_{\varrho}(y)} \frac{|u(y)-u(z)|}{d(y, z)} ;
$$

we shall denote by $c_{P}$ the minimal constant verifying the Poincaré inequality. We write the integral average of a function $u$ over a ball $B_{r}(x)$ as $u_{B_{r}(x)}$. Let us mention in passing that, by the doubling property, for every $B_{R}(x) \subset X$ and $y \in B_{R}(x)$ and for $0<r \leq R<\infty$ the inequality

$$
\frac{\mu\left(B_{R}(x)\right)}{\mu\left(B_{r}(y)\right)} \leq C\left(\frac{R}{r}\right)^{q_{\mu}},
$$

holds, where $C$ is a positive constant depending only on $c_{D}$ and $q_{\mu}=\log _{2} c_{D}$. In what follows, $q_{\mu}$ denotes a counterpart of dimension related to the measure $\mu$ on $X$.

Unless otherwise stated, $C$ will denote a positive constant whose exact value is not important and it may change even within a line. A concentric $\alpha$-dilate, $\alpha>1$, of a ball $B=B_{r}(x)=B(x, r)$ is written as $\alpha B$ or $B_{\alpha r}(x)$. We write the topological boundary of a set $E$ as $\partial E$, and $\partial^{*} E$ denotes the measure theoretic boundary of $E$, i.e. the set of points $x \in X$ where both $E$ and its complement $X \backslash E$ have positive upper density. Recall that $\partial^{*} E \subset \partial E$.

Finally, by the Hausdorff measure of co-dimension one we mean the generalized spherical Hausdorff measure $\mathcal{H}$ obtained by applying the Carathéodory construction to the function $h\left(B_{r}(x)\right)=\mu\left(B_{r}(x)\right) / r($ see $[\mathrm{A}])$. 
2.2. Differentiable structure. An upper gradient for an extended real-valued function $u$ on $X$ is a Borel function $g: X \rightarrow[0, \infty]$ such that

$$
\left|u(\gamma(0))-u\left(\gamma\left(l_{\gamma}\right)\right)\right| \leq \int_{\gamma} g d s
$$

for every nonconstant compact rectifiable curve $\gamma:\left[0, l_{\gamma}\right] \rightarrow X$. This notion is due to [HK]. We say that $g$ is a $p$-weak upper gradient of $u$ if (2.2) holds for $p$-almost every curve, see $[\mathrm{KM}]$. If $u$ has an upper gradient in $L^{p}(X)$, then there exists a unique minimal $p$-weak upper gradient $g_{u} \in L^{p}(X)$ of $u$, where $g_{u} \leq g \mu$-a.e. for every $p$-weak upper gradient $g \in L^{p}(X)$ of $u$.

Under our standing assumptions on $X$, we have also the Cheeger differentiable structure available (see $[\mathrm{C}]$ ) that allow us to define a linear gradient operator for Lipschitz functions. There exists a countable measurable partition $U_{\alpha}$ of $X$, and Lipschitz coordinate charts $X^{\alpha}=\left(X_{1}^{\alpha}, \ldots, X_{k_{\alpha}}^{\alpha}\right): X \rightarrow \mathbb{R}^{k_{\alpha}}$ such that $\mu\left(U_{\alpha}\right)>0$ for each $\alpha$, and $\mu\left(X \backslash \bigcup_{\alpha} U_{\alpha}\right)=0$. Moreover, for all $\alpha$ the charts $\left(X_{1}^{\alpha}, \ldots, X_{k_{\alpha}}^{\alpha}\right)$ are linearly independent on $U_{\alpha}$ and $1 \leq k_{\alpha} \leq N$, where $N$ is a constant depending on $c_{D}, c_{P}$, and $\lambda$, and in addition for any Lipschitz function $u: X \rightarrow \mathbb{R}$ there is an associated unique (up to a set of zero $\mu$-measure) measurable function $D_{\alpha} u: U_{\alpha} \rightarrow \mathbb{R}^{k_{\alpha}}$ for which the following Taylor-type approximation

$$
u(x)=u\left(x_{0}\right)+D_{\alpha} u\left(x_{0}\right) \cdot\left(X_{\alpha}(x)-X_{\alpha}\left(x_{0}\right)\right)+o\left(d\left(x, x_{0}\right)\right)
$$

holds for $\mu$-a.e. $x_{0} \in U_{\alpha}$. In particular, for $x \in U_{\alpha}$ there exists a norm $\|\cdot\|_{x}$ on $\mathbb{R}^{k_{\alpha}}$ equivalent to the Euclidean norm $|\cdot|$, such that $g_{u}(x)=\left\|D_{\alpha} u(x)\right\|_{x}$ for almost every $x \in U_{\alpha}$. Moreover, it is possible to show that there exists a constant $c>1$ such that $c^{-1} g_{u}(x) \leq|D u(x)| \leq c g_{u}(x)$ for all Lipschitz functions $u$ and $\mu$-a.e. $x \in X$. By $D u(x)$ we mean $D_{\alpha} u(x)$ whenever $x \in U_{\alpha}$. Indeed, one can choose the cover such that $U_{\alpha} \cap U_{\beta}$ is empty whenever $\alpha \neq \beta$.

For the definition of the Sobolev spaces $N^{1, p}(X)$ we will follow [Sh]. Since we assume $X$ to satisfy a 1 -Poincaré inequality, the Sobolev space $N^{1, p}(X), 1 \leq p<\infty$, can also be defined as the closure of the collection of Lipschitz functions on $X$ in the $N^{1, p}$-norm defined as $\|u\|_{1, p}^{p}=\|u\|_{L^{p}(X)}^{p}+\left\|g_{u}\right\|_{L^{p}(X)}^{p}$. The space $N^{1, p}(X)$ equipped with the $N^{1, p}$ norm is a Banach space and a lattice $[\mathrm{Sh}]$. By $[\mathrm{FHK}]$, the Cheeger differentiable structure extends to all functions in $N^{1, p}(X)$.

2.3. Semigroup associated with $\mathcal{E}$. In the metric space setting, there is a standard way to construct a semigroup by using the Dirichlet forms approach. The best way to construct it is to use the $L^{2}$-theory of (bilinear) Dirichlet forms and then apply a classical result asserting that such semigroup can be extrapolated to any $L^{p}, 1 \leq p \leq \infty$.

We start with the following Dirichlet form $\mathcal{E}: L^{2}(X) \times L^{2}(X) \rightarrow[-\infty, \infty]$ defined in terms of the Cheeger differentiable structure by

$$
\mathcal{E}(u, v)=\int_{X} D u(x) \cdot D v(x) d \mu(x)
$$

with domain $D(\mathcal{E}):=N^{1,2}(X)$ (if $u$ or $v$ does not belong to $N^{1,2}(X)$, then $\mathcal{E}(u, v)=\infty$ ). This bilinear form is an example of a regular and strongly local Dirichlet form as defined 
in [FOT]. The associated infinitesimal generator $A$ acts on a dense subspace $D(A)$ of $N^{1,2}(X)$ so that for each $u \in D(A)$ and for every $v \in N^{1,2}(X)$,

$$
\int_{X} v A u d \mu=-\mathcal{E}(u, v)
$$

The operator $A$ is dissipative in the sense that

$$
\int_{X} u A u d \mu=-\mathcal{E}(u, u) \leq 0
$$

and is merely the Laplacian $\Delta$ when $X=\mathbb{R}^{n}$, the Cheeger differentiable structure is the standard Euclidean structure, and $\mu$ is the Lebesgue measure.

We also point out that under our standing assumptions, the metric induced by the form

$$
d_{\varepsilon}(x, y)=\sup \left\{f(x)-f(y): f \in N^{1,2}(X),|D f| \leq 1 \mu \text {-a.e. }\right\}
$$

is bi-Lipschitz equivalent to the original metric $d$. Note that the Cheeger differential structure satisfies $|D f|$ is comparable to the minimal 2-weak upper gradient of $f$. Therefore, if $|D f| \leq 1 \mu$-a.e. in $X$ then $f$ has a Lipschitz continuous representative.

Remark 2.1. Associated with the above Dirichlet form $\mathcal{E}$ and its infinitesimal generator A there is a Markov semigroup $\left(T_{t}\right)_{t>0}$ acting on $L^{2}(X)$ with the following properties (we refer to $[\mathrm{FOT}],[\mathrm{KRS}]$, or $[\mathrm{MMS}]$ for properties (1) through (7)):

(1) $T_{t} \circ T_{s}=T_{t+s}$ for all $t, s>0$;

(2) since $A$ is symmetric in $L^{2}(X)$, then $T_{t}$ is self adjoint in $L^{2}(X)$, that is

$$
\int_{X} T_{t} f g d \mu=\int_{X} f T_{t} g d \mu
$$

for all $f, g \in L^{2}(X)$;

(3) $T_{t} f \rightarrow f$ in $L^{2}(X)$ when $t \rightarrow 0$;

(4) since $A$ is dissipative, $T_{t}$ is a contraction in $L^{2}(X)$, i.e. $\left\|T_{t} f\right\|_{L^{2}(X)} \leq\|f\|_{L^{2}(X)}$ for all $f \in L^{2}(X)$ and $t>0$;

(5) if $f \in D(A)$, then $\frac{1}{t}\left(T_{t} f-f\right) \rightarrow$ Af in $L^{2}(X)$ as $t \rightarrow 0$;

(6) for all $t>0$ and $f \in L^{2}(X)$, we have that $\partial_{t} T_{t} f$ and $A T_{t} f$ are both in $L^{2}(X)$ and

$$
\partial_{t} T_{t} f=A T_{t} f
$$

(7) $\left(T_{t}\right)_{t>0}$ is a Markovian semigroup, that is if $0 \leq f \leq 1$, then $0 \leq T_{t} f \leq 1$;

(8) $\left(T_{t}\right)_{t>0}$ can be extended to $L^{\infty}(X)$ by first considering positive functions $f \geq 0$ and sequences $f_{n} \in L^{2}(X), f_{n} \nearrow f$ and setting

$$
T_{t} f=\lim _{n \rightarrow \infty} T_{t} f_{n}
$$

we refer to [FOT, p. 56];

(9) since the measure $\mu$ is doubling, $\left(T_{t}\right)_{t>0}$ is stochastically complete : $T_{t} 1=1$ [St1, Theorem 4] (see also $[\mathrm{G}]$ for stochastic completeness in the manifold setting);

(10) Since $\left|T_{t} f\right| \leq T_{t}|f| \mu$-a.e. for $f \in L^{1}(X) \cap L^{\infty}(X)$, and since $L^{1}(X) \cap L^{\infty}(X)$ is dense in $L^{1}(X),\left(T_{t}\right)_{t}$ can be extended to a contraction semigroup on $L^{1}(X)$. 
A measurable function $p: \mathbb{R} \times X \times X \rightarrow[0, \infty]$ is called a heat kernel or transition function on $X$ associated with the semigroup $\left(T_{t}\right)_{t>0}$ if

$$
T_{t} f(x)=\int_{X} p(t, x, y) f(y) d \mu(y),
$$

for every $f \in L^{2}(X)$ and for every $t>0$. For $t \leq 0$ we have $p(t, x, y)=0$, and $p(t, x, y)=p(t, y, x)$ by the symmetry of the semigroup. The existence of a heat kernel is a direct consequence of the linearity of the operator $f \mapsto T_{t} f$ together with the $L^{\infty}$ boundedness of such an operator (Markovian property) and the Riesz representation theorem, see for instance Sturm [St2, Proposition 2.3]. Standard regularity arguments on doubling metric measure spaces admitting a 2-Poincaré inequality imply that the map $x \mapsto p(t, x, y)$ is Hölder continuous for any $(t, y) \in(0, \infty) \times X$.

Under our standing assumptions on $X$, we have the following estimates for a heat kernel uniformly for all $x, y \in X$ and all $t>0$, we refer to [St3, Corollary 4.10]. There are positive constants $C, C_{1}, C_{2}$ such that

$$
\begin{aligned}
& p(t, x, y) \geq \frac{C^{-1} e^{-\frac{d(x, y)^{2}}{C_{1} t}}}{\sqrt{\mu\left(B_{\sqrt{t}}(x)\right)} \sqrt{\mu\left(B_{\sqrt{t}}(y)\right)}}, \\
& p(t, x, y) \leq \frac{C e^{-\frac{d(x, y)^{2}}{C_{2} t}}}{\sqrt{\mu\left(B_{\sqrt{t}}(x)\right)} \sqrt{\mu\left(B_{\sqrt{t}}(y)\right)}} .
\end{aligned}
$$

2.4. BV functions and sets of finite perimeter. Following [Mr], we say that a function $u \in L^{1}(X)$ is of bounded variation $(u \in \mathrm{BV}(X))$ if

$$
\|D u\|(X)=\inf \left\{\liminf _{j \rightarrow \infty} \int_{X} g_{u_{j}} d \mu: u_{j} \in \operatorname{Lip}_{\mathrm{loc}}(X), u_{j} \rightarrow u \text { in } L_{\mathrm{loc}}^{1}(X)\right\}
$$

is finite, where $g_{u_{j}}$ is the minimal 1-weak upper gradient of $u_{j}$. Moreover, a Borel set $E \subset X$ is said to have finite perimeter if $\chi_{E} \in \mathrm{BV}(X)$. We denote the perimeter measure of $E$ by $P(E)=\left\|D \chi_{E}\right\|(X)$. By replacing $X$ with an open set $F$ we may define $\|D u\|(F)$ and we shall write the perimeter measure of $E$ with respect to $F$ as $P(E, F)=\left\|D \chi_{E}\right\|(F)$.

Strictly speaking, the definition given in $[\mathrm{Mr}]$ considers $\operatorname{lip}\left(u_{j}\right)$ instead of $g_{u_{j}}$. However, under our standing assumptions of doubling property and 1-Poincaré inequality, $\operatorname{lip}\left(u_{j}\right)=g_{u_{j}} \mu$-a.e. in $X$ (see $[\mathrm{C}]$ or $\left.[\mathrm{HKST}]\right)$.

An equivalent definition can be also given by way of the Cheeger differentiable structure by replacing a 1 -weak upper gradient of $u_{j}$ with its Cheeger derivative. We shall say that $u$ has bounded total Cheeger variation if $\left\|D_{c} u\right\|(X)<\infty$. A set with Cheeger finite perimeter is a $\mu$-measurable set $E$ such that $\left\|D_{c} \chi_{E}\right\|(X)<\infty$. By the results contained in $[\mathrm{C}]$, it follows that these two definitions are equivalent, in the sense that $C^{-1}\left\|D_{c} u\right\| \leq\|D u\| \leq C\left\|D_{c} u\right\|$. For further equivalent definitions of $\|D u\|$, perhaps more fruitful in a metric setting where Poincaré inequality and doubling properties may not be available, see $[A D]$. 
It follows from a 1-Poincaré inequality that for each $u \in \mathrm{BV}(X)$

$$
\int_{B_{r}(x)}\left|u(y)-u_{B_{r}(x)}\right| d \mu(y) \leq c_{P} r\|D u\|\left(B_{2 \lambda r}(x)\right) .
$$

The factor of 2 in the ball on the right-hand side follows from the fact that weak limits of measures might charge the boundary of $B_{\lambda r}(x)$. In particular, if $E$ is a set of finite perimeter and $u=\chi_{E}$ we recover the following form of the relative isoperimetric inequality

$$
\mu\left(B_{r}(x) \cap E\right) \frac{\mu\left(B_{r}(x) \backslash E\right)}{\mu\left(B_{r}(x)\right)} \leq c_{P} r P\left(E, B_{2 \lambda r}(x)\right) .
$$

From the proof of [BH, Theorem 1.1] (see Section 3 of $[\mathrm{BH}]$ ) and the above 1-Poincaré inequality, we have the following regularity for sets of finite perimeter. Given a Lipschitz function $f$ on $X$, for $t \in \mathbb{R}$ we set $A_{t}=\{x \in X: f(x)>t\}$. Then for almost every $t \in \mathbb{R}$ we have that

$$
\liminf _{r \rightarrow 0} \frac{\mu\left(\bigcup_{x \in \partial A_{t}} B_{r}(x)\right)}{r} \leq C P\left(A_{t}\right)<\infty .
$$

Next, we recall that for any $u \in \mathrm{BV}(X)$ and Borel set $E \subset X$ the co-area formula

$$
\int_{-\infty}^{\infty} P(\{x \in X: u(x)>t\}, E) d t=\|D u\|(E)
$$

holds, and for the proof, we refer to [Mr, Proposition 4.2].

Finally, we recall that the measure $P(E, F)$ is concentrated on the set

$$
\Sigma_{\gamma}=\left\{x: \limsup _{r \rightarrow 0} \min \left\{\frac{\mu\left(B_{r}(x) \cap E\right)}{\mu\left(B_{r}(x)\right)}, \frac{\mu\left(B_{r}(x) \backslash E\right)}{\mu\left(B_{r}(x)\right)}\right\} \geq \gamma\right\} \subset \partial^{*} E,
$$

where constant $\gamma>0$ depends only on $c_{D}, c_{P}$ and $\lambda$, and moreover $\mathcal{H}\left(\partial^{*} E \backslash \Sigma_{\gamma}\right)=0$ and $\mathcal{H}\left(\partial^{*} E\right)<\infty$. We refer the reader to [A, Theorem 5.3, Theorem 5.4].

\section{A Characterization of BV FUnCtions}

In the setting of metric measure spaces satisfying the standing assumptions listed in Section 2, we now give a new characterization of BV functions in terms of the neardiagonal parts of the Korevaar-Schoen type energy [KS]. The product measure $\mu \otimes \mu$ in the space $X \times X$ shall be written as $\mu(x, y)$.

We point out here that in the more general setting of topological spaces $X$, with $X \times X$ equipped with a nonnegative symmetric Radon measure $\Gamma$ locally finite outside the diagonal, Maz'ya proved in $[\mathrm{M}]$ a conductor inequality for compactly supported continuous functions $f$ on $X$ for which

$$
\langle f\rangle_{p, \Gamma}^{p}:=\int_{X \times X}|f(x)-f(y)|^{p} d \Gamma(x, y)<\infty .
$$


It was shown in [M, Theorem 2, Section 4] that, when $1 \leq p \leq q<\infty$ and $a>1$, the following co-area-type integral can be majorized by the energy $\langle f\rangle_{p, \Gamma}$

$$
\left(\int_{0}^{\infty} \operatorname{cap}_{p, \Gamma}\left(\bar{M}_{a t}, M_{t}\right)^{q / p} d t^{q}\right)^{p / q} \leq C\langle f\rangle_{p, \Gamma}^{p}
$$

where $M_{t}=\{x \in X:|f(x)|>t\}$ and the capacity is obtained via minimization of $\langle\varphi\rangle_{p, \Gamma}^{p}$ over all admissible functions $\varphi$. With $p=q=1$, in general the constant $C$ in (3.1) depends on $a$, and blows up in the order of $(a-1)^{-1}$ as $a \rightarrow 1$, see [M].

If we now take $\Gamma_{a}$ to be defined by

$$
d \Gamma_{a}(x, y)=\frac{\chi_{\Delta^{a-1}}(x, y) d \mu(x, y)}{(a-1) \sqrt{\mu\left(B_{a-1}(x)\right)} \sqrt{\mu\left(B_{a-1}(y)\right)}},
$$

we obtain a near-diagonal energy

$$
\langle f\rangle_{1, \Gamma_{a}}=\frac{1}{a-1} \int_{\Delta^{a-1}} \frac{|f(x)-f(y)|}{\sqrt{\mu\left(B_{a-1}(x)\right)} \sqrt{\mu\left(B_{a-1}(y)\right)}} d \mu(x, y),
$$

with the constant $C$ in (3.1) now independent of $a$. Here $\Delta^{\varepsilon}, \varepsilon>0$, denotes the $\varepsilon$ neighborhood of the diagonal in $X \times X$, i.e.

$$
\Delta^{\varepsilon}=\{(x, y) \in X \times X: d(x, y)<\varepsilon\} .
$$

We point out here that when $p=q=1$, the above Maz'ya-type inequality, (3.1), in this setting, is equivalent to the the following generalization of the co-area inequality (with $\varepsilon=a-1)$

$$
\limsup _{\varepsilon \rightarrow 0} \frac{1}{\varepsilon} \int_{0}^{\infty}\left(\int_{M_{t}} \int_{B_{\varepsilon}(x) \backslash M_{t}} \frac{d \mu(y) d \mu(x)}{\sqrt{\mu\left(B_{\varepsilon}(x)\right)} \sqrt{\mu\left(B_{\varepsilon}(y)\right)}}\right) d t \leq C \limsup _{\varepsilon \rightarrow 0}\langle f\rangle_{1, \Gamma_{1+\varepsilon}}
$$

associated with the near-diagonal Korevaar-Schoen energy.

In this section we will show that in our more specialized setting of metric measure spaces, the family (with respect to $\varepsilon>0$ ) of above energies $\langle f\rangle_{1, \Gamma_{1+\varepsilon}}$ corresponding to a given function $f$ has a finite limit infimum as $\varepsilon \rightarrow 0$ if and only if $f$ is in the BV class. The proof will also show that for small $\varepsilon>0$ the above energy is controlled by a constant multiple of the BV-energy of $f$. This also provides a connection between the energy studied by Maz'ya [M] in our, more specialized, setting and the BV-energy.

Let us now formulate the main theorem of this section.

Theorem 3.1. Suppose that $u \in L^{1}(X)$. Then $u \in \mathrm{BV}(X)$ if, and only if,

$$
\liminf _{\varepsilon \rightarrow 0^{+}} \frac{1}{\varepsilon} \int_{\Delta^{\varepsilon}} \frac{|u(x)-u(y)|}{\sqrt{\mu\left(B_{\varepsilon}(x)\right)} \sqrt{\mu\left(B_{\varepsilon}(y)\right)}} d \mu(x, y)<\infty .
$$

Remark 3.2. As can be deduced from the proof, we in fact will show that if $u \in B V(X)$ or if the limit in the above theorem is finite, then the limit supremum of the expression 
considered in the theorem is comparable with $|D u|$, that is, there is a constant $C \geq 1$, that depends only on the data of the space $X$, such that

$$
C^{-1}\|D u\|(X) \leq \liminf _{\varepsilon \rightarrow 0^{+}} \frac{1}{\varepsilon} \int_{\Delta_{\varepsilon}} \frac{|u(x)-u(y)|}{\sqrt{\mu\left(B_{\varepsilon}(x)\right)} \sqrt{\mu\left(B_{\varepsilon}(y)\right)}} d \mu(x, y) \leq C\|D u\|(X) .
$$

Proof of Theorem 3.1. Let us assume first that $u \in \mathrm{BV}(X)$ and fix $\varepsilon>0$. Then we can find a sequence of points in $X,\left\{x_{i}\right\}_{i \in \mathbb{N}}$, such that

$$
X=\bigcup_{i \in \mathbb{N}} B_{\varepsilon}\left(x_{i}\right) \text {, and } B_{\varepsilon / 2}\left(x_{i}\right) \cap B_{\varepsilon / 2}\left(x_{j}\right)=\emptyset \text { whenever } i \neq j,
$$

and such that the covering has bounded overlap

$$
\sum_{i \in \mathbb{N}} \chi_{B_{4 \lambda \varepsilon}\left(x_{i}\right)}(x) \leq c_{O}
$$

Since for $x \in B_{\varepsilon}\left(x_{i}\right)$ and $y \in B_{\varepsilon}(x)$ we have that $B_{\varepsilon}\left(x_{i}\right) \subset B_{2 \varepsilon}(x)$ and $B_{\varepsilon}\left(x_{i}\right) \subset B_{4 \varepsilon}(y)$, we get, by the doubling property of $\mu$,

$$
\begin{aligned}
& \mu\left(B_{\varepsilon}\left(x_{i}\right)\right) \leq \mu\left(B_{4 \varepsilon}(y)\right) \leq c_{D}^{2} \mu\left(B_{\varepsilon}(y)\right), \\
& \mu\left(B_{\varepsilon}\left(x_{i}\right)\right) \leq \mu\left(B_{2 \varepsilon}(x)\right) \leq c_{D} \mu\left(B_{\varepsilon}(x)\right) .
\end{aligned}
$$

We obtain

$$
\begin{aligned}
& \int_{\Delta^{\varepsilon}} \frac{|u(x)-u(y)|}{\sqrt{\mu\left(B_{\varepsilon}(x)\right)} \sqrt{\mu\left(B_{\varepsilon}(y)\right)}} d \mu(x, y) \\
& \leq c_{D}^{\frac{3}{2}} \sum_{i \in \mathbb{N}} \frac{1}{\mu\left(B_{\varepsilon}\left(x_{i}\right)\right)} \int_{B_{\varepsilon}\left(x_{i}\right)} \int_{B_{\varepsilon}(x)}|u(x)-u(y)| d \mu(y) d \mu(x) \\
& \leq c_{D}^{\frac{3}{2}} \sum_{i \in \mathbb{N}} \frac{1}{\mu\left(B_{\varepsilon}\left(x_{i}\right)\right)} \int_{B_{\varepsilon}\left(x_{i}\right)} \int_{B_{\varepsilon}(x)}\left|u(x)-u_{B_{\varepsilon}\left(x_{i}\right)}\right| d \mu(y) d \mu(x) \\
& \quad+c_{D}^{\frac{3}{2}} \sum_{i \in \mathbb{N}} \frac{1}{\mu\left(B_{\varepsilon}\left(x_{i}\right)\right)} \int_{B_{\varepsilon}\left(x_{i}\right)} \int_{B_{\varepsilon}(x)}\left|u(y)-u_{B_{\varepsilon}\left(x_{i}\right)}\right| d \mu(y) d \mu(x) \\
& =C\left(I_{1}+I_{2}\right)
\end{aligned}
$$

Since $B_{\varepsilon}(x) \subset B_{2 \varepsilon}\left(x_{i}\right)$ for $x \in B_{\varepsilon}\left(x_{i}\right)$ we get, by a 1-Poincaré inequality,

$$
\begin{aligned}
I_{1} & =\sum_{i \in \mathbb{N}} \frac{1}{\mu\left(B_{\varepsilon}\left(x_{i}\right)\right)} \int_{B_{\varepsilon}\left(x_{i}\right)} \mu\left(B_{\varepsilon}(x)\right)\left|u(x)-u_{B_{\varepsilon}\left(x_{i}\right)}\right| d \mu(x) \\
& \leq \sum_{i \in \mathbb{N}} \frac{\mu\left(B_{2 \varepsilon}\left(x_{i}\right)\right)}{\mu\left(B_{\varepsilon}\left(x_{i}\right)\right)} \int_{B_{\varepsilon}\left(x_{i}\right)}\left|u(x)-u_{B_{\varepsilon}\left(x_{i}\right)}\right| d \mu(x) \\
& \leq \varepsilon c_{P} c_{D} \sum_{i \in \mathbb{N}}\|D u\|\left(B_{2 \lambda \varepsilon}\left(x_{i}\right)\right) \leq \varepsilon C\|D u\|(X) .
\end{aligned}
$$


We treat the term $I_{2}$ in a similar fashion

$$
\begin{aligned}
& I_{2} \leq \sum_{i \in \mathbb{N}} \frac{1}{\mu\left(B_{\varepsilon}\left(x_{i}\right)\right)} \int_{B_{\varepsilon}\left(x_{i}\right)} d \mu(x) \int_{B_{2 \varepsilon}\left(x_{i}\right)}\left|u(y)-u_{B_{\varepsilon}\left(x_{i}\right)}\right| d \mu(y) \\
& =\sum_{i \in \mathbb{N}} \int_{B_{2 \varepsilon}\left(x_{i}\right)}\left|u(y)-u_{B_{\varepsilon}\left(x_{i}\right)}\right| d \mu(y) \\
& \leq \sum_{i \in \mathbb{N}} \int_{B_{2 \varepsilon}\left(x_{i}\right)}\left|u(y)-u_{B_{2 \varepsilon}\left(x_{i}\right)}\right| d \mu(y) \\
& \quad+\sum_{i \in \mathbb{N}}\left|u_{B_{\varepsilon}\left(x_{i}\right)}-u_{B_{2 \varepsilon}\left(x_{i}\right)}\right| \mu\left(B_{2 \varepsilon}\left(x_{i}\right)\right) \\
& \leq 2 \varepsilon c_{P}\left(1+c_{D}\right) \sum_{i \in \mathbb{N}}\|D u\|\left(B_{4 \lambda \varepsilon}\left(x_{i}\right)\right) \leq \varepsilon C\|D u\|(X),
\end{aligned}
$$

where we have used a 1-Poincaré inequality and the fact that

$$
\begin{aligned}
\mu\left(B_{2 \varepsilon}\left(x_{i}\right)\right) \mid u_{B_{\varepsilon}\left(x_{i}\right)}-u_{B_{2 \varepsilon}\left(x_{i}\right)} & \leq C \int_{B_{\varepsilon}\left(x_{i}\right)}\left|u(x)-u_{B_{2 \varepsilon}\left(x_{i}\right)}\right| d \mu(x) \\
& \leq C \int_{B_{2 \varepsilon}\left(x_{i}\right)}\left|u(x)-u_{B_{2 \varepsilon}\left(x_{i}\right)}\right| d \mu(x) \\
& \leq \varepsilon C\|D u\|\left(B_{4 \lambda \varepsilon}\left(x_{i}\right)\right) .
\end{aligned}
$$

This completes the proof of the claim that if $u \in \mathrm{BV}(X)$ then the limit infimum is finite (in fact, we have obtained that the limsup is finite).

For the converse statement, we assume that

$$
\liminf _{\varepsilon \rightarrow 0^{+}} \frac{1}{\varepsilon} \int_{\Delta^{\varepsilon}} \frac{|u(x)-u(y)|}{\sqrt{\mu\left(B_{\varepsilon}(x)\right)} \sqrt{\mu\left(B_{\varepsilon}(y)\right)}} d \mu(x, y)<\infty .
$$

For every $\varepsilon>0$, let us define a positive and finite measure on $X$ as

$$
d \mu_{\varepsilon}(x)=\widetilde{u}_{B_{\varepsilon}}(x) d \mu(x)
$$

where for each $x \in X$ we set

$$
\widetilde{u}_{B_{\varepsilon}}(x)=\frac{1}{\mu\left(B_{\varepsilon}(x)\right)} \int_{B_{\varepsilon}(x)} \frac{|u(x)-u(y)|}{\varepsilon} d \mu(y) .
$$

Note that

$$
\int_{X} \tilde{u}_{\varepsilon}(x) d \mu(x) \leq \frac{C}{\varepsilon} \int_{\Delta_{\varepsilon}} \frac{|u(x)-u(y)|}{\sqrt{\mu\left(B_{\varepsilon}(x)\right)} \sqrt{\mu\left(B_{\varepsilon}(y)\right)}} d \mu(x, y),
$$

and so we have that

$$
\lim _{\varepsilon \rightarrow 0^{+}} \int_{X} \tilde{u}_{\varepsilon}(x) d \mu(x) \leq \liminf _{\varepsilon \rightarrow 0^{+}} \frac{C}{\varepsilon} \int_{\Delta_{\varepsilon}} \frac{|u(x)-u(y)|}{\sqrt{\mu\left(B_{\varepsilon}(x)\right)} \sqrt{\mu\left(B_{\varepsilon}(y)\right)}} d \mu(x, y) .
$$

Let $\left\{B_{i}^{\varepsilon}=B_{\varepsilon}\left(x_{i}\right)\right\}$ be a family of balls which covers $X$ with the following bounded overlap property:

$$
\sum_{i \in \mathbb{N}} \chi_{B_{6 \varepsilon}\left(x_{i}\right)}(x) \leq c_{O}
$$


Then, with respect to this family of balls, there exists a collection $\left(\varphi_{i}^{\varepsilon}\right)_{i}$ of functions on $X$ such that each $\varphi_{i}^{\varepsilon}$ is $C \varepsilon^{-1}$-Lipschitz continuous, where $C$ is a constant depending only on the doubling constant $c_{D}, 0 \leq \varphi_{i}^{\varepsilon} \leq 1$ for all $i, \varphi_{i}^{\varepsilon}(x)=0$ for $x \in X \backslash B_{i}^{2 \varepsilon}$ for every $i$, and $\sum_{i} \varphi_{i}^{\varepsilon}(x)=1$ for all $x \in X$. The collection $\left(\varphi_{i}^{\varepsilon}\right)_{i}$ forms a partition of unity of $X$ with respect to $\left\{B_{i}^{\varepsilon}\right\}$. We refer the reader to [HKT], and the references therein, for these properties of $\varphi_{i}^{\varepsilon}$. Let us then define

$$
u_{\varepsilon}(x)=\sum_{i=1}^{\infty} u_{B_{i}^{\varepsilon}} \varphi_{i}^{\varepsilon}(x)
$$

for every $x \in X$. The function $u_{\varepsilon}$ is locally Lipschitz continuous, since it is locally a finite sum of Lipschitz functions. Furthermore, the sequence $\left(u_{\varepsilon}\right)_{\varepsilon}$ converges to $u$ in $L^{1}(X)$ as $\varepsilon \rightarrow 0$ [HKT, Lemma 5.3.(2)].

We need an estimate on the Lipschitz constant of $u_{\varepsilon}$. Suppose that $x, y \in B_{i}^{\varepsilon}$ and let $J=\left\{j: B_{i}^{2 \varepsilon} \cap B_{j}^{2 \varepsilon} \neq \emptyset\right\}$. By the doubling property, balls $B_{i}^{\varepsilon}$ have bounded overlap and so the cardinality of $J$ is bounded by a constant depending only on the doubling constant $c_{D}$, that is $\# J \leq c_{\#}$ with $c_{\#}=c_{\#}\left(c_{D}\right)$. In addition $B_{j}^{\varepsilon} \subset B_{i}^{6 \varepsilon}$. By the properties of the partition of unity, we have exactly as in [HKT] that for any $x, y \in B_{i}^{\varepsilon}$

$$
\begin{aligned}
\left|u_{\varepsilon}(x)-u_{\varepsilon}(y)\right| & =\left|\sum_{j \in J}\left(u_{B_{j}^{\varepsilon}}-u_{B_{i}^{\varepsilon}}\right)\left(\varphi_{j}^{\varepsilon}(x)-\varphi_{j}^{\varepsilon}(y)\right)\right| \\
& \leq C \frac{d(x, y)}{\varepsilon} \sum_{j \in J}\left|u_{B_{i}^{\varepsilon}}-u_{B_{j}^{\varepsilon}}\right| .
\end{aligned}
$$

For every $j \in J$ we also have that

$$
\begin{aligned}
\left|u_{B_{i}^{\varepsilon}}-u_{B_{j}^{\varepsilon}}\right| & \leq \frac{1}{\mu\left(B_{i}^{\varepsilon}\right) \mu\left(B_{j}^{\varepsilon}\right)} \int_{B_{i}^{\varepsilon}} \int_{B_{j}^{\varepsilon}}|u(y)-u(x)| d \mu(y) d \mu(x) \\
& \leq \frac{1}{\mu\left(B_{i}^{\varepsilon}\right) \mu\left(B_{j}^{\varepsilon}\right)} \int_{B_{i}^{\varepsilon}} \int_{B_{6 \varepsilon}(x)}|u(y)-u(x)| d \mu(y) d \mu(x) \\
& \leq \frac{\varepsilon C}{\mu\left(B_{i}^{\varepsilon}\right)} \mu_{6 \varepsilon}\left(B_{i}^{\varepsilon}\right),
\end{aligned}
$$

where in the last inequality we have used the fact that $B_{j}^{\varepsilon} \subset B_{6 \varepsilon}(x)$ whenever $x \in B_{i}^{\varepsilon}$. In other words, we have proved that

$$
\operatorname{lip}\left(u_{\varepsilon}\right)(x) \leq C \frac{c_{\#}}{\mu\left(B_{i}^{\varepsilon}\right)} \mu_{6 \varepsilon}\left(B_{i}^{\varepsilon}\right)
$$

for every $x \in B_{i}^{\varepsilon}$. Here, $\mu_{6 \varepsilon}$ is as in (3.3). We therefore obtain

$$
\begin{aligned}
\int_{X} \operatorname{lip}\left(u_{\varepsilon}\right)(x) d \mu(x) & \leq \sum_{i \in \mathbb{N}} \int_{B_{i}^{\varepsilon}} \operatorname{lip}\left(u_{\varepsilon}\right)(x) d \mu(x) \leq C \sum_{i=1}^{\infty} c_{\#} \mu_{6 \varepsilon}\left(B_{i}^{\varepsilon}\right) \\
& \leq C \mu_{6 \varepsilon}(X) .
\end{aligned}
$$


Now, by the assumption and the doubling property of the measure $\mu$, we have by (3.4)

$$
\begin{aligned}
\liminf _{\varepsilon \rightarrow 0^{+}} \mu_{\varepsilon}(X) & =\liminf _{\varepsilon \rightarrow 0^{+}} \int_{X} \tilde{u}_{B_{\varepsilon}}(x) d \mu(x) \\
& \leq C \liminf _{\varepsilon \rightarrow 0^{+}} \frac{1}{\varepsilon} \int_{\Delta_{\varepsilon}} \frac{|u(x)-u(y)|}{\sqrt{\mu\left(B_{\varepsilon}(x)\right)} \sqrt{\mu\left(B_{\varepsilon}(y)\right)}} d \mu(y, x)<\infty,
\end{aligned}
$$

and hence we can find a sequence $\left\{\varepsilon_{j}\right\}_{j}$ going to 0 such that

$$
\sup _{j \in \mathbb{N}} \mu_{\varepsilon_{j}}(X)<\infty
$$

and then the sequence of Lipschitz functions $u_{j}=u_{\varepsilon_{j} / 6}$ converges to $u$ in $L^{1}(X)$ and

$$
\begin{aligned}
\|D u\|(X) & \leq \limsup _{j \rightarrow \infty} \int_{X} \operatorname{lip}\left(u_{j}\right)(x) d \mu(x) \\
& \leq C \liminf _{\varepsilon \rightarrow 0^{+}} \frac{1}{\varepsilon} \int_{\Delta_{\varepsilon}} \frac{|u(x)-u(y)|}{\sqrt{\mu\left(B_{\varepsilon}(x)\right)} \sqrt{\mu\left(B_{\varepsilon}(y)\right)}} d \mu(y, x)<\infty,
\end{aligned}
$$

which implies that $u \in \mathrm{BV}(X)$.

\section{Sets of Finite Perimeter: A Ledoux type Characterization}

We shall make use of Theorem 3.1 to connect the sets of finite perimeter to the theory of heat semigroup in the sense of Ledoux [L]. The reader could also consult $[\mathrm{P}]$.

The Ledoux characterization in the limit of (1.1) requires global information of the decay of the heat extension. So if the space is hyperbolic then the behavior of $E$ and $\mathbb{R}^{n} \backslash E$ far away from $\partial E$ also might play a role in the limit given in (1.1). We therefore modify the criterion and consider only regions near $\partial E$.

Theorem 4.1. Let $E \subset X$ be $\mu$-measurable and assume that $E$ has finite measure. Then we have the following.

(1) If

$$
\liminf _{t \rightarrow 0^{+}} \frac{1}{\sqrt{t}} \int_{E^{\sqrt{t}} \backslash E} T_{t} \chi_{E}(x) d \mu(x)<\infty,
$$

then $E$ is of finite perimeter.

(2) If $E$ is of finite perimeter and satisfies the inequality

$$
\liminf _{r \rightarrow 0} \frac{\mu\left(\bigcup_{x \in \partial E} B_{r}(x)\right)}{r} \leq C P(E),
$$

then $E$ satisfies (4.1).

Here, $E^{\sqrt{t}}$ is the tubular neighborhood

$$
E^{\sqrt{t}}=\bigcup_{x \in E} B_{\sqrt{t}}(x)
$$


Furthermore, every set $E$ of finite perimeter can be approximated in the relaxed sense by open sets of finite perimeter that satisfy inequality (4.2), that is, we can find a sequence of sets $E_{k}$ of finite perimeter satisfying (4.2) such that $\chi_{E_{k}} \rightarrow \chi_{E}$ in $L^{1}(X)$ and

$$
P(E) \leq \lim _{k \rightarrow \infty} \liminf _{t \rightarrow 0^{+}} \frac{1}{\sqrt{t}} \int_{E^{\sqrt{t}} \backslash E} T_{t} \chi_{E_{k}}(x) d \mu(x) \leq C P(E) .
$$

We remark that unlike in the case of Theorem 3.1, see Remark 3.2, here we do not have equivalence between the expression in (4.1) and the perimeter $P(E)$ of $E$. We only obtain that

$$
P(E) \leq C \liminf _{t \rightarrow 0^{+}} \frac{1}{\sqrt{t}} \int_{E^{\sqrt{t}} \backslash E} T_{t} \chi_{E}(x) d \mu(x)
$$

Proof of Theorem 4.1. Note first that

$$
\begin{aligned}
\int_{X}\left|T_{t} \chi_{E}(x)-\chi_{E}(x)\right| d \mu(x) & \\
= & \int_{X}\left|\int_{X} p(t, x, y)\left(\chi_{E}(y)-\chi_{E}(x)\right) d \mu(y)\right| d \mu(x) \\
= & \int_{E}\left|-\int_{X \backslash E} p(t, x, y) d \mu(y)\right| d \mu(x) \\
& +\int_{X \backslash E}\left|\int_{E} p(t, x, y) d \mu(y)\right| d \mu(x) \\
= & 2 \int_{E} \int_{X \backslash E} p(t, x, y) d \mu(x) d \mu(y) \\
= & 2 \int_{X} \int_{X} \chi_{X \backslash E}(x) p(t, x, y) \chi_{E}(y) d \mu(x) d \mu(y) \\
= & 2 \int_{X \backslash E} T_{t} \chi_{E}(x) d \mu(x) .
\end{aligned}
$$

Hence by the fact that $T_{t} f \rightarrow f$ in $L^{1}(X)$ as $t \rightarrow 0$ for each $f \in L^{1}(X)$, we have that

$$
\lim _{t \rightarrow 0^{+}} \int_{X \backslash E} T_{t} \chi_{E}(x) d \mu(x)=0 .
$$

Suppose now that in addition,

$$
\liminf _{t \rightarrow 0^{+}} \frac{1}{\sqrt{t}} \int_{E^{\sqrt{t}} \backslash E} T_{t} \chi_{E}(x) d \mu(x)<\infty
$$


We want to show that the set $E$ has finite perimeter. By the symmetry of the heat kernel

$$
\begin{gathered}
2 \int_{E} \int_{E^{\sqrt{t}} \backslash E} p(t, x, y) d \mu(y) d \mu(x) \geq \int_{E} \int_{B_{\sqrt{t}}(x) \backslash E} p(t, x, y) d \mu(y) d \mu(x) \\
+\int_{E \sqrt{t} \backslash E} \int_{B_{\sqrt{t}}(x) \cap E} p(t, x, y) d \mu(y) d \mu(x) \\
=\int_{\Delta \sqrt{t}} p(t, x, y)\left|\chi_{E}(y)-\chi_{E}(x)\right| d \mu \times \mu(y, x)
\end{gathered}
$$

To obtain the last equality above, we used the fact that $\left|\chi_{E}(x)-\chi_{E}(y)\right|=1$ precisely when

$$
(x, y) \in E \times(X \backslash E) \cup(X \backslash E) \times E,
$$

and zero otherwise.

By estimate (2.3) for the kernel $p(t, x, y)$,

$$
\begin{aligned}
\frac{1}{\sqrt{t}} \int_{E} \int_{E^{\sqrt{t}} \backslash E} p(t, & x, y) d \mu(y) d \mu(x) \\
& \geq \frac{C}{\sqrt{t}} \int_{\Delta^{\sqrt{t}} \cap\left[\left(E^{\sqrt{t}} \cap E\right) \times\left(E^{\sqrt{t}} \backslash E\right)\right]} \frac{\left|\chi_{E}(x)-\chi_{E}(y)\right|}{\sqrt{\mu\left(B_{\sqrt{t}}(x)\right)} \sqrt{\mu\left(B_{\sqrt{t}}(y)\right)}} d \mu(y) d \mu(x) \\
& =\frac{C}{\sqrt{t}} \int_{\Delta \sqrt{t}} \frac{\left|\chi_{E}(x)-\chi_{E}(y)\right|}{\sqrt{\mu\left(B_{\sqrt{t}}(x)\right)} \sqrt{\mu\left(B_{\sqrt{t}}(y)\right)}} d \mu(y) d \mu(x),
\end{aligned}
$$

and so we have

$$
\liminf _{t \rightarrow 0^{+}} \frac{1}{\sqrt{t}} \int_{\Delta \sqrt{t}} \frac{\left|\chi_{E}(x)-\chi_{E}(y)\right|}{\sqrt{\mu\left(B_{\sqrt{t}}(x)\right)} \sqrt{\mu\left(B_{\sqrt{t}}(y)\right)}} d \mu(y) d \mu(x)<\infty .
$$

By Theorem 3.1, we conclude that $\chi_{E} \in \mathrm{BV}(X)$. This completes the proof of statement (1) of the theorem.

To prove statement (2) of the theorem, suppose that $E \subset X$ is open and of finite perimeter, with $\mu(E)$ finite, and that (4.2) is satisfied by $E$.

For each $t>0$ let

$$
E^{\sqrt{t}} \backslash E \subset \bigcup_{k \geq 0} B_{k}
$$

where $B_{k}:=B_{2 \sqrt{t}}\left(x_{k}\right)$ with $x_{k} \in \partial E$ such that the dilated balls $2 \lambda B_{k}$ have bounded overlap (3.2), the bound $c_{O}$ of the overlap depending solely on the doubling constant of the measure $\mu$. For each $k \geq 0$, we also write $E=E_{k}^{1} \cup E_{k}^{2}$, where

$$
E_{k}^{1}=E \cap B_{k}, \quad E_{k}^{2}=E \backslash B_{k} .
$$


Then

$$
\begin{aligned}
\frac{1}{\sqrt{t}} \int_{E \sqrt{t} \backslash E} T_{t} \chi_{E}(x) d \mu(x) & =\frac{1}{\sqrt{t}} \int_{E} \int_{E^{\sqrt{t}} \backslash E} p(t, x, y) d \mu(y) d \mu(x) \\
& \leq \frac{1}{\sqrt{t}} \sum_{k=0}^{\infty} \int_{E} \int_{B_{k} \backslash E} p(t, x, y) d \mu(y) d \mu(x) \\
& \leq \frac{1}{\sqrt{t}} \sum_{k=0}^{\infty}\left(\int_{E_{k}^{1}} \int_{B_{k} \backslash E} p(t, x, y) d \mu(y) d \mu(x)\right. \\
& \left.+\int_{E_{k}^{2}} \int_{B_{k} \backslash E} p(t, x, y) d \mu(y) d \mu(x)\right) \\
& =: \frac{1}{\sqrt{t}} \sum_{k=0}^{\infty}\left(I_{k}^{1}+I_{k}^{2}\right) .
\end{aligned}
$$

Let us estimate the preceding terms separately, starting with the term $I_{k}^{1}$. By (2.4), the doubling condition and (2.1), and finally by (2.5), we have

$$
\begin{aligned}
I_{k}^{1} & =\int_{E \cap B_{k}} \int_{B_{k} \backslash E} p(t, x, y) d \mu(y) d \mu(x) \leq \int_{E \cap B_{k}} \int_{B_{k} \backslash E} \frac{C}{\mu\left(B_{\sqrt{t}}(x)\right)} d \mu(y) d \mu(x) \\
& \leq C \frac{\mu\left(B_{k} \backslash E\right)}{\mu\left(B_{k}\right)} \mu\left(E \cap B_{k}\right) \leq C \sqrt{t} P\left(E, 2 \lambda B_{k}\right) .
\end{aligned}
$$

Therefore, we may conclude that

$$
\frac{1}{\sqrt{t}} \sum_{k=0}^{\infty} I_{k}^{1} \leq C P(E)
$$

We point out here again that $C$ represents constants that depend only on the data of $X$ and whose particular value we do not care about, and that the value of $C$ could change even within a line.

The second term $I_{k}^{2}$ is treated as follows. We set

$$
E_{k}^{2}=\bigcup_{j \geq 1}\left[\bar{B}_{2^{j+1} \sqrt{t}}\left(x_{k}\right) \backslash B_{2^{j} \sqrt{t}}\left(x_{k}\right)\right] \cap E=: \bigcup_{j \geq 0} A_{k}^{j} .
$$

By (2.4) we have

$$
\begin{aligned}
I_{k}^{2} & =\int_{E \backslash B_{k}} \int_{B_{k} \backslash E} p(t, x, y) d \mu(y) d \mu(x) \\
& \leq C \int_{B_{k} \backslash E} \sum_{j=1}^{\infty} \int_{A_{k}^{j}} \frac{e^{-C 4^{j}}}{\sqrt{\mu\left(B_{k}\right)} \sqrt{\mu\left(B_{\sqrt{t}}(y)\right)}} d \mu(y) d \mu(x) \\
& \leq C \frac{\mu\left(B_{k} \backslash E\right)}{\sqrt{\mu\left(B_{k}\right)}} \sum_{j=1}^{\infty} \int_{A_{k}^{j}} \frac{e^{-C 4^{j}}}{\sqrt{\mu\left(B_{\sqrt{t}}(y)\right)}} d \mu(y) .
\end{aligned}
$$


By (2.1) we know that whenever $y \in B_{2^{j+1} \sqrt{t}}\left(x_{k}\right)$,

$$
\frac{\mu\left(B_{\sqrt{t}}(y)\right)}{\mu\left(B_{2^{j+1} \sqrt{t}}\left(x_{k}\right)\right)} \geq C\left(\frac{\sqrt{t}}{2^{j+1} \sqrt{t}}\right)^{q_{\mu}}=C 2^{-j q_{\mu}} .
$$

Therefore,

$$
\begin{aligned}
I_{k}^{2} & \leq C \frac{\mu\left(B_{k} \backslash E\right)}{\sqrt{\mu\left(B_{k}\right)}} \sum_{j=1}^{\infty} \int_{A_{k}^{j}} \frac{e^{-C 4^{j}} 2^{j q_{\mu} / 2}}{\sqrt{\mu\left(B_{2^{j+1} \sqrt{t}}\left(x_{k}\right)\right)}} d \mu(y) \\
& \leq C \frac{\mu\left(B_{k} \backslash E\right)}{\sqrt{\mu\left(B_{k}\right)}} \sum_{j=1}^{\infty} \frac{e^{-C 4^{j}} 2^{j q_{\mu} / 2}}{\sqrt{\mu\left(B_{2^{j+1} \sqrt{t}}\left(x_{k}\right)\right)}} \mu\left(A_{k}^{j}\right) \\
& \leq C \frac{\mu\left(B_{k} \backslash E\right)}{\sqrt{\mu\left(B_{k}\right)}} \sum_{j=1}^{\infty} e^{-C 4^{j}} 2^{j q_{\mu} / 2} \sqrt{\mu\left(B_{2^{j+1} \sqrt{t}}\left(x_{k}\right)\right)} .
\end{aligned}
$$

By (2.1),

$$
\mu\left(B_{2^{j+1} \sqrt{t}}\left(x_{k}\right)\right) \leq C 2^{j q_{\mu}} \mu\left(B_{\sqrt{t}}\left(x_{k}\right)\right)=C 2^{j q_{\mu}} \mu\left(B_{k}\right)
$$

It follows that

$$
I_{k}^{2} \leq C \frac{\mu\left(B_{k} \backslash E\right)}{\sqrt{\mu\left(B_{k}\right)}} \sum_{j=0}^{\infty} e^{-C 4^{j}} 2^{j q_{\mu}} \sqrt{\mu\left(B_{k}\right)} \leq C \mu\left(B_{k} \backslash E\right)
$$

Therefore, by the bounded overlap of the balls $B_{k}$,

$$
\frac{1}{\sqrt{t}} \sum_{k=0}^{\infty} I_{k}^{2} \leq C \frac{\mu\left(E^{\sqrt{t}} \backslash E\right)}{\sqrt{t}}
$$

and the fact that (4.2) holds for $E$, completes the proof of the statement (2) of the theorem.

Finally, the last claim of the theorem follows from the proof of [BH, Theorem 1.1] (see Section 3 of $[\mathrm{BH}]$ ), see also [KKST]. This completes the proof of the theorem.

Remark 4.2. The estimates of $\sum_{k=0}^{\infty} I_{k}^{1}$ and $\sum_{k=0}^{\infty} I_{k}^{2}$ hold also for all open sets $E$ of finite perimeter. However, while for all open sets $E$ of finite perimeter we do have that

$$
\liminf _{t \rightarrow 0^{+}} \frac{1}{\sqrt{t}} \sum_{k=0}^{\infty} I_{k}^{1} \leq C P(E)<\infty
$$

to know that

$$
\liminf _{t \rightarrow 0^{+}} \frac{1}{\sqrt{t}} \sum_{k=0}^{\infty} I_{k}^{2} \leq C P(E)
$$

we need equation (4.2). Hence, our requirements for the set $E$ in claim (2) of the theorem. For example, in $\mathbb{R}^{2}$ the set

$$
E=\bigcup_{k \geq 0} B_{2^{-k}}\left(q_{k}\right)
$$


with $\left\{q_{k}\right\}_{k \geq 0}$ an enumeration of $\mathbb{Q} \times \mathbb{Q}$ is an open set of finite perimeter, but $\mu\left(E^{\sqrt{t}} \backslash E\right)=$ $\infty$ for all $t>0$.

This may merely be an artifact of our method of proof. Note that in $\mathbb{R}^{n}$, an alternative proof due to Bakry shows that the additional requirement (4.2) is not needed to obtain the Ledoux-type characterization (4.1) of sets of finite perimeter. We do not know how to adapt the proof of Bakry in such a generality considered in our paper.

\section{Total variation: De Giorgi characterization under Bakry-Émery CONDITION}

We close this paper by proving a metric space version of the De Giorgi characterization $[\mathrm{DeG}]$ of the total variation of a BV function. Our approach is based on recent works by Amborsio, Gigli, and Savaré [AGS] and is essentially a consequence of [S].

We refer to [CM, MP, GP, BMP] for the De Giorgi characterization on Riemannian manifolds and on Carnot groups, and to [AMPP] for the same result but with the semigroup of a rather general second order elliptic operator on domains in Euclidean spaces. All of these results require a condition on the curvature of the spaces; these conditions are related to the Ricci curvature in the case of Riemannian manifolds. In $[\mathrm{MP}]$ the requirement was on a lower bound of Ricci curvature plus a technical requirement on a lower bound on the volume of balls. It was pointed out in $[\mathrm{CM}]$ that, thanks to the works of Bakry and Émery (see for instance [BE]), the same result can be obtained only with a lower bound condition on the Ricci curvature. In $[\mathrm{GP}]$ it has been shown that one can also require that the Ricci tensor can be split into two parts, one bounded below and one belonging to a Kato class. Also in this case the De Giorgi characterization of the total variation holds true.

We recall the definition of $\mathrm{BE}(K, \infty)$ condition as formulated in $[\mathrm{BE}, \mathrm{Ba}]$.

Definition 5.1 (Bakry-Émery condition). The Dirichlet form $\mathcal{E}$ given in Section 2.3 in relation to the Cheeger differentiable structure is said to satisfy the $\mathrm{BE}(K, \infty)$ condition, $K \in \mathbb{R}$, if for every $f \in D(A)$ such that $A f \in N^{1,2}(X)$, we have

$$
\frac{1}{2} \int_{X}|D f|^{2} A \varphi d \mu-\int_{X} \varphi D f \cdot D A f d \mu \geq K \int_{X} \varphi|D f|^{2} d \mu
$$

whenever $\varphi \in D(A) \cap L^{\infty}(X)$ is nonnegative such that $A \varphi \in L^{\infty}(X)$.

We remind the reader about the connections between the $\operatorname{BE}(K, \infty)$ condition and the doubling property and a Poincaré inequality assumed in the previous sections. It is known that a 1-Poincaré inequality follows from the $\operatorname{BE}(K, \infty)$ condition, or even from weaker curvature conditions like the $\operatorname{CD}(K, \infty)$ condition of Lott-Sturm-Villani. However, a complete separable metric space endowed with a probability measure and satisfying the $\operatorname{BE}(K, \infty)$ condition need not be doubling.

In the appendix, we recall some consequences of the Bakry-Émery condition. These consequences are needed in the proof of the main result of this section, Proposition 5.2, and have been investigated for instance in $[\mathrm{S}]$. The setting in [S] is rather general, and hence for the convenience of the reader we provide sketches of simplified proofs of these results in the appendix. 
The main result of this section is the following.

Proposition 5.2. Let $u \in L^{1}(X)$. Suppose that

$$
\limsup _{t \rightarrow 0^{+}} \int_{X}\left|D T_{t} u\right| d \mu<\infty
$$

then $u \in \mathrm{BV}(X)$. Here, by assuming that $u$ satisfies (5.2), we are also implicitly assuming that $T_{t} u \in N^{1,1}(X)$ for each $t>0$. On the other hand, whenever (5.2) holds and if a Dirichlet form $\mathcal{E}$ compatible with the differentiable structure satisfies the $\mathrm{BE}(K, \infty)$ condition, we have

$$
\left\|D_{c} u\right\|(X)=\lim _{t \rightarrow 0^{+}} \int_{X}\left|D T_{t} u\right| d \mu .
$$

By the self-improvement property of the Bakry-Émery condition $\mathrm{BE}(K, \infty)$, we can easily prove Proposition 5.2. Of course, it would be interesting to obtain a similar result, or even the weaker version as in $[\mathrm{BMP}]$, without imposing the Bakry-Émery condition on $\mathcal{E}$.

Proof of Proposition 5.2. The claim that (5.2) implies that $u \in \mathrm{BV}(X)$ follows immediately from the definition of $\mathrm{BV}(X)$ upon noticing that as $u \in L^{1}(X)$ we must have $T_{t} u \rightarrow u$ in $L^{1}(X)$ as $t \rightarrow 0$ (see Remark 2.1(10)) and $T_{t} u \in N^{1,1}(X)$. Furthermore, it is also immediate that

$$
\left\|D_{c} u\right\|(X) \leq \limsup _{t \rightarrow 0^{+}} \int_{X}\left|D T_{t} u\right| d \mu .
$$

Let $u \in \mathrm{BV}(X)$. We consider a sequence of Lipschitz functions $\left(f_{j}\right)_{j \in \mathbb{N}} \subset \operatorname{Lip}_{c}(X)$ such that $f_{j} \rightarrow u$ in $L^{1}(X)$ and

$$
\left\|D_{c} u\right\|(X)=\lim _{j \rightarrow \infty} \int_{X}\left|D f_{j}\right| d \mu .
$$

By the lower semicontinuity of the total variation together with Proposition 6.3 and the fact that $\left(T_{t}\right)_{t>0}$ is a contraction semigroup on $L^{1}(X)$ (see Remark 2.1(10)), we conclude

$$
\begin{aligned}
\left\|D_{c} u\right\|(X) \leq & \liminf _{t \rightarrow 0^{+}} \int_{X}\left|D T_{t} u\right| d \mu \leq \liminf _{t \rightarrow 0^{+}} \liminf _{j \rightarrow \infty} \int_{X}\left|D T_{t} f_{j}\right| d \mu \\
& \leq \liminf _{t \rightarrow 0^{+}} \liminf _{j \rightarrow \infty} e^{-K t} \int_{X} T_{t}\left|D f_{j}\right| d \mu \\
& \leq \liminf _{t \rightarrow 0^{+}} \liminf _{j \rightarrow \infty} e^{-K t} \int_{X}\left|D f_{j}\right| d \mu=\left\|D_{c} u\right\|(X),
\end{aligned}
$$

and hence the proof is complete.

In conclusion, we note that by the analog of both the De Giorgi characterization and the Ledoux characterization of functions of bounded variation demonstrated in this paper, even in the general metric measure space setting (with the measure doubling and supporting a 1-Poincaré inequality), the behavior of sets of finite perimeter is intimately connected to the heat semigroup. 


\section{Appendix}

In this appendix we gather together properties associated with the Bakry-Émery condition in Section 5. In the metric setting a related condition, guaranteed by the logarithmic Sobolev inequality, was first studied in [KRS]. The results in this appendix are from $[\mathrm{S}]$ adapted to our purposes. Given that we have access to the Cheeger differential structure related to the Dirichlet form, some of the arguments in [S] are simplified in our setting.

In the sequel, the following Pazy convolution operator will play an important role:

$$
\beta_{\varepsilon} f=\frac{1}{\varepsilon} \int_{0}^{\infty} T_{s} f \varrho(s / \varepsilon) d s .
$$

Here $\varrho \in C_{c}^{\infty}(0, \infty)$ is a nonnegative convolution kernel with $\int_{0}^{\infty} \varrho(s) d s=1$. We also define the measure operator $A^{*}$ by setting

$$
\begin{aligned}
& D\left(A^{*}\right)=\left\{f \in N^{1,2}(X): \text { there exists } \nu \in \mathcal{M}(X)\right. \text { such that } \\
& \left.\qquad \mathcal{E}(f, v)=-\int_{X} v d \nu \text { for all } v \in \operatorname{Lip}_{c}(X)\right\},
\end{aligned}
$$

and denote by $A^{*} f$ the measure $\nu$. Here $\mathcal{M}(X)$ denotes the set of finite Borel measures on $X$. The integration by parts formula

$$
\int_{X} D f \cdot D \varphi d \mu=-\int_{X} \varphi d A^{*} f
$$

can be extended from functions $\varphi \in \operatorname{Lip}_{c}(X)$ to functions $\varphi \in N^{1,2}(X) \cap L^{\infty}(X)$ if on the right hand side we consider the representative $\tilde{\varphi} \in N^{1,2}(X)$ of $\varphi$, since $A^{*} f$ does not charge sets of zero Sobolev 2-capacity. We refer to [MMS] for more details. Since $\mu$ is doubling and supports a 2-Poincaré inequality, and because $X$ is assumed to be complete, it follows that Lipschitz functions with compact support form a dense subclass of $N^{1,2}(X)$ (see [Sh] for example).

We consider the function class

$$
\mathbb{D}_{\infty}=\left\{f \in D(A) \cap \operatorname{Lip}_{b}(X): A f \in N^{1,2}(X)\right\} ;
$$

as we shall see in Proposition 6.1, if $f \in \mathbb{D}_{\infty}$, then $|D f|^{2} \in D\left(A^{*}\right)$. We shall denote by $\Gamma_{2}^{*}(f)$ the measure

$$
\Gamma_{2}^{*}(f)=\frac{1}{2} A^{*}|D f|^{2}-(D f \cdot D A f) \mu=\Gamma_{2}^{\perp}(f)+\gamma_{2}(f) \mu
$$

where $\Gamma_{2}^{\perp}(f) \perp \mu$ is the singular part of the measure $\Gamma_{2}^{*}(f)$ and $\gamma_{2}(f)$ its absolute continuous component.

We list in the following proposition the main properties of functions in $\mathbb{D}_{\infty}$ needed in the proof of Proposition 5.2. We refer to [S] for the details of the proof, but we provide a sketch of the proof for the convenience of the reader.

Proposition 6.1. Suppose that $f \in \mathbb{D}_{\infty}$ and that $\mathcal{E}$ satisfies the $\mathrm{BE}(K, \infty)$ condition. Then

(1) $|D f|^{2} \in N^{1,2}(X)$; 
(2) $|D f|^{2} \in D\left(A^{*}\right)$ and for any nonnegative $\varphi \in \operatorname{Lip}_{c}(X)$,

$$
\frac{1}{2} \int_{X} \varphi d A^{*}|D f|^{2} d \mu-\int_{X} \varphi D f \cdot D A f d \mu \geq K \int_{X} \varphi|D f|^{2} d \mu
$$

(3) we have

$$
\left.\left.\int_{X} \varphi|D| D f\right|^{2}\right|^{2} d \mu \leq 4 \int_{X} \varphi\left(\gamma_{2}(f)-K|D f|^{2}\right) d \mu
$$

for any nonnegative $\varphi \in \operatorname{Lip}_{c}(X)$.

Proof. Let us prove (1). Since $f \in \mathbb{D}_{\infty}$, condition $\operatorname{BE}(K, \infty)$ implies that

$$
\int_{X}|D f|^{2} A \varphi d \mu \geq 2 \int_{X}\left(K|D f|^{2}+D f \cdot D A f\right) \varphi d \mu
$$

for any positive $\varphi \in D(A)$. Then, by setting $u=|D f|^{2}$ and $u_{\varepsilon}=\beta_{\varepsilon} u$, taking into account the fact that for any $\varphi \in D(A)$, since $A$ commutes with the Pazy convolution $\beta_{\epsilon}$, there holds

$$
\int_{X} u_{\varepsilon} A \varphi d \mu=\int_{X} u A \beta_{\epsilon} \varphi d \mu
$$

We obtain

$$
\begin{aligned}
\int_{X}\left|D u_{\epsilon}\right|^{2} d \mu & =-\int_{X} u_{\epsilon} A u_{\epsilon} d \mu=-\int_{X} u A \beta_{\epsilon} u_{\epsilon} d \mu \\
& \leq-2 \int_{X}\left(K|D f|^{2}+D f \cdot D A f\right) \beta_{\epsilon} u_{\epsilon} d \mu .
\end{aligned}
$$

Passing to the limit as $\varepsilon \rightarrow 0$, we arrive at the conclusion that $|D f|^{2} \in N^{1,2}(X)$ with

$$
\left.\left.\int_{X}|D| D f\right|^{2}\right|^{2} d \mu \leq-2 \int_{X}\left(K|D f|^{2}+D f \cdot D A f\right)|D f|^{2} d \mu .
$$

Let us then prove (2). Using the $\mathrm{BE}(K, \infty)$ condition and by approximating any $\operatorname{Lip}_{c}(X)$ function with functions in $D(A)$, we can deduce that

$$
\int_{X} A u_{\varepsilon} \varphi d \mu \geq-\int_{X} g \beta_{\epsilon} \varphi d \mu=-\int_{X} \beta_{\varepsilon} g \varphi d \mu
$$

for any $\varphi \in \operatorname{Lip}_{c}(X)$, where we have defined

$$
g=-2\left(K|D f|^{2}+D f \cdot D A f\right)
$$

Passing to the limit as $\varepsilon \rightarrow 0$, we define

$$
\mathcal{L}_{f}(\varphi):=-\int_{X} D|D f|^{2} \cdot D \varphi d \mu+\int_{X} g \varphi d \mu
$$

which is a positive linear functional defined on $\operatorname{Lip}_{c}(X)$. If in the estimate

$$
0 \leq \int_{X}\left(A u_{\varepsilon}+\beta_{\epsilon} g\right) \varphi d \mu
$$


we take an increasing sequence of Lipschitz functions $\varphi_{n}$ such that $0 \leq \varphi_{n} \leq 1, \varphi_{n} \equiv 1$ on $B_{n}\left(x_{0}\right)$ and $\varphi_{n} \equiv 0$ on $X \backslash B_{n+1}\left(x_{0}\right)$ for a fixed point $x_{0}$, by the dominated convergence theorem,

$$
0 \leq \int_{X}\left(A u_{\varepsilon}+\beta_{\varepsilon} g\right) \varphi_{n} d \mu \leq \int_{X}\left(A u_{\varepsilon}+\beta_{\varepsilon} g\right) d \mu=\int_{X} \beta_{\varepsilon} g d \mu=\int_{X} g d \mu .
$$

Note that

$$
\int_{X} A u_{\varepsilon} d \mu=\int_{X} \chi_{X} A u_{\varepsilon} d \mu=-\int_{X} D u \cdot D \chi_{X} d \mu=0
$$

since $D \chi_{X}=0$. We also note that

$$
\left|A u_{\varepsilon}\right| \leq \frac{1}{\varepsilon} \int_{s_{\varepsilon}}^{S_{\varepsilon}}\left|A T_{s}(u)\right| \rho(s / \varepsilon) d s \leq C_{\varepsilon} \int_{s_{\varepsilon}}^{S_{\varepsilon}}\left|A T_{s}(u)\right| d s,
$$

and, therefore, $A u_{\varepsilon}$ is in $L^{1}(X)$.

We have obtained

$$
\left|\mathcal{L}_{f}(\varphi)\right| \leq\|\varphi\|_{\infty} \int_{X} g d \mu
$$

and then $\mathcal{L}_{f}$ can be represented by a positive measure $\lambda \in \mathcal{M}(X)$. This proves that $|D f|^{2} \in D\left(A^{*}\right)$ with

$$
A^{*}|D f|^{2}=\lambda-g \mu
$$

The positivity of the measure

$$
\frac{1}{2} A^{*}|D f|^{2}-\left(D f \cdot D A f+K|D f|^{2}\right) \mu
$$

follows by the positivity of $\lambda$.

The proof of (3) is rather technical and does not simplify in our setting, so we refer to [S, Theorem 3.4].

The Bakry-Émery condition in Definition 5.1 has equivalent formulations, we refer to $[\mathrm{W}]$ for the Riemannian case and to [AGS, S] for the metric space setting. We recall here some of those equivalent formulations that we shall use.

Proposition 6.2. The following are equivalent:

(1) E satisfies the $\mathrm{BE}(K, \infty)$ condition;

(2) for any $f \in N^{1,2}(X)$, any $t>0$, and for every nonnegative $\varphi \in \operatorname{Lip}_{c}(X)$

$$
\int_{X} \varphi\left|D T_{t} f\right|^{2} d \mu \leq e^{-2 K t} \int_{X} \varphi T_{t}|D f|^{2} d \mu
$$

(3) for any $f \in L^{2}(X)$, any $t>0$, and for every nonnegative $\varphi \in \operatorname{Lip}_{c}(X)$

$$
\frac{e^{2 K t}-1}{K} \int_{X} \varphi\left|D T_{t} f\right|^{2} d \mu \leq \int_{X} \varphi\left(T_{t} f^{2}-\left(T_{t} f\right)^{2}\right) d \mu .
$$

Proof. Let us show that (1) implies (2). Let $f \in N^{1,2}(X)$ and $\varphi \in D(A) \cap L^{\infty}(X)$ such that $A \varphi \in L^{\infty}(X)$ and $\varphi \geq 0$ be fixed. We define

$$
G_{\varphi}(s)=\int_{X} T_{s} \varphi\left|D T_{t-s} f\right|^{2} d \mu .
$$


Taking derivatives, and by $\operatorname{BE}(K, \infty)$, we get

$$
\begin{aligned}
G_{\varphi}^{\prime}(s) & =\int_{X} A T_{s} \varphi\left|D T_{t-s} f\right|^{2} d \mu-2 \int_{X} T_{s} \varphi D T_{t-s} f \cdot D A T_{t-s} f d \mu \\
& \geq 2 K \int_{X} T_{s} \varphi\left|D T_{t-s} f\right|^{2} d \mu=2 K G_{\varphi}(s) .
\end{aligned}
$$

If for some $s>0, G_{\varphi}(s)=0$, then if $\varphi$ is not identically zero, $T_{s} \varphi>0$ and we must have $\left|D T_{t-s} f\right|=0$ at $\mu$-a.e. Hence $T_{t-s} f$ is constant and thus $f$ is also constant. In this case (2) is trivially satisfied. On the other hand, if $G_{\varphi}(s)>0$, we can integrate and conclude that

$$
G_{\varphi}(t) \geq G_{\varphi}(0) e^{2 K t}
$$

which is condition (2).

Let us show that (2) implies (3). By introducing the function

$$
G_{\varphi}(s)=\int_{X} T_{s} \varphi\left(T_{t-s} f\right)^{2} d \mu
$$

taking derivatives, and taking into account (2), we obtain

$$
\begin{aligned}
G_{\varphi}^{\prime}(s) & =\int_{X} A T_{s} \varphi\left(T_{t-s} f\right)^{2} d \mu-2 \int_{X} T_{s} \varphi T_{t-s} f A T_{t-s} f d \mu=2 \int_{X} \varphi T_{s}\left|D T_{t-s} f\right|^{2} d \mu \\
& \geq 2 e^{2 K s} \int_{X} \varphi\left|D T_{s} T_{t-s} f\right|^{2} d \mu=2 e^{2 K s} \int_{X} \varphi\left|D T_{t} f\right|^{2} d \mu .
\end{aligned}
$$

By integrating with respect to $s$, we obtain condition (3).

Let us now assume (3) and let us take $f \in D(A)$ such that $A f \in N^{1,2}(X)$ and $\varphi \in$ $D(A) \cap L^{\infty}(X)$ with $A \varphi \in L^{\infty}$. We can define the function

$$
F_{\varphi}(t)=\int_{X} \varphi\left(T_{t} f^{2}-\left(T_{t} f\right)^{2}\right) d \mu .
$$

The second order Taylor expansion formula implies

$$
F_{\varphi}(t)=2 t \int_{X} \varphi|D f|^{2} d \mu+t^{2}\left(\int_{X}|D f|^{2} A \varphi d \mu+2 \int_{X} \varphi D f \cdot D A f d \mu\right)+o\left(t^{2}\right) .
$$

In the same way, we obtain that

$$
\begin{aligned}
G_{\varphi}(t) & =\frac{e^{2 K t}-1}{K} \int_{X} \varphi\left|D T_{t} f\right|^{2} d \mu \\
& =2 t \int_{X} \varphi|D f|^{2} d \mu+2 t^{2}\left(K \int_{X} \varphi|D f|^{2} d \mu+2 \int_{X} \varphi D f \cdot D A f d \mu\right)+o\left(t^{2}\right) .
\end{aligned}
$$

Then, since

$$
\begin{aligned}
0 & \leq F_{\varphi}(t)-G_{\varphi}(t) \\
& =2 t^{2}\left(\int_{X} A \varphi|D f|^{2} d \mu-2 \int_{X} \varphi D f \cdot D A f d \mu-2 K \int_{X} \varphi|D f| d \mu\right)+o\left(t^{2}\right)
\end{aligned}
$$

we obtain condition (1). 
Under the above hypotheses, we can prove the following self-improving result of the Bakry-Émery condition. The result is essentially contained in a monograph by Bakry [Bakry], see also Deuschel and Strook [DS, Lemma 6.2.39], in the Riemannian setting. The self-improvement of the Bakry-Émery condition has also been obtained by Savaré in [S, Corollary 3.5] in very general setting. We provide a proof here for the sake of completeness.

Proposition 6.3. Suppose that a Dirichlet form $\mathcal{E}$ compatible with the differentiable structure satisfies the $\operatorname{BE}(K, \infty)$ condition for some $K \in \mathbb{R}$. Then for any $f \in N^{1,2}(X)$, any $t>0$, and every nonnegative $\varphi \in \operatorname{Lip}_{c}(X)$, we have

$$
\int_{X} \varphi\left|D T_{t} f\right| d \mu \leq e^{-K t} \int_{X} \varphi T_{t}|D f| d \mu .
$$

Proof. We start by considering $f \in \mathbb{D}_{\infty}$; we point out that by $(6.2), \mathbb{D}_{\infty}$ is dense in $N^{1,2}(X)$. Indeed, $\operatorname{Lip}_{b}(X)$ is dense in $N^{1,2}(X)$ and taking approximations of functions $f \in N^{1,2}(X) \cap \operatorname{Lip}_{b}(X)$ in terms of the semigroup, we get that $T_{t} f \in \mathbb{D}_{\infty}$ for any $t>0$ by $(6.2)$ and

$$
A T_{t} f=T_{t / 2} A T_{t / 2} f \in D(A) \subset N^{1,2}(X) .
$$

Here we also used the fact that by the Bakry-Émery condition (6.2), if $f \in \operatorname{Lip}_{b}(X)$ then $T_{t} f \in \operatorname{Lip}_{b}(X)$.

Let us fix $\delta>0$ and set

$$
u_{t}^{\delta}(x):=\sqrt{\left|D T_{t} f(x)\right|^{2}+\delta^{2}}-\delta .
$$

We also fix a nonnegative function $\varphi \in N^{1,2}(X) \cap L^{\infty}(X)$ and introduce the function

$$
G_{\delta}(s)=\int_{X} T_{s} \varphi u_{t-s}^{\delta} d \mu
$$

If we take the derivative, we obtain:

$$
\begin{aligned}
& G^{\prime}(s)=\int_{X} A T_{s} \varphi u_{t-s}^{\delta} d \mu-\int_{X} \frac{T_{s} \varphi}{u_{t-s}^{\delta}+\delta} D T_{t-s} f \cdot D A T_{t-s} f d \mu \\
&=-\int_{X} \frac{1}{2\left(u_{t-s}^{\delta}+\delta\right)} D T_{s} \varphi \cdot D\left|D T_{t-s} f\right|^{2} d \mu-\int_{X} \frac{T_{s} \varphi}{u_{t-s}^{\delta}+\delta} D T_{t-s} f \cdot D A T_{t-s} f d \mu \\
&= \int_{X} \frac{T_{s} \varphi}{2\left(u_{t-s}^{\delta}+\delta\right)} d A^{*}\left|D T_{t-s} f\right|^{2}-\left.\left.\frac{1}{4} \int_{X} \frac{T_{s} \varphi}{\left(u_{t-s}^{\delta}+\delta\right)^{3}}|D| D T_{t-s} f\right|^{2}\right|^{2} d \mu \\
&= \int_{X} \frac{T_{s} \varphi}{u_{t-s}^{\delta}+\delta} d \Gamma_{2}^{*}\left(T_{t-s} f\right)-\left.\left.\frac{1}{4} \int_{X} \frac{T_{s} \varphi}{\left(u_{t-s}^{\delta}+\delta\right)^{3}}|D| D T_{t-s} f\right|^{2}\right|^{2} d \mu \\
& \geq \int_{X} \frac{T_{s} \varphi}{u_{t-s}^{\delta}+\delta} \gamma_{2}\left(T_{t-s} f\right) d \mu \\
& \quad-\int_{X} \frac{T_{s} \varphi}{\left(u_{t-s}^{\delta}+\delta\right)^{3}}\left|D T_{t-s} f\right|^{2}\left(\gamma_{2}\left(T_{t-s} f\right)-K\left|D T_{t-s} f\right|^{2}\right) d \mu,
\end{aligned}
$$


where in the last line we have used the properties contained in Proposition 6.1. We have

$$
\begin{aligned}
G_{\delta}^{\prime}(s) & \geq \int_{X} \frac{T_{s} \varphi}{\left(u_{t-s}^{\delta}+\delta\right)^{3}}\left(\delta^{2} \gamma_{2}\left(T_{t-s} f\right)-K \delta^{2}\left|D T_{t-s} f\right|^{2}\right. \\
& \left.+K\left|D T_{t-s} f\right|^{2}\left(u_{t-s}^{\delta}+\delta\right)^{2}\right) d \mu \\
& \geq K \int_{X} \frac{T_{s} \varphi}{u_{t-s}^{\delta}+\delta}\left|D T_{t-s} f\right|^{2} d \mu \geq K \int_{X} T_{s} \varphi u_{t-s}^{\delta} d \mu,
\end{aligned}
$$

where we used the fact that $\gamma_{2}(f)-K|D f|^{2} \geq 0 \mu$-a.e. by Proposition 6.1. We thus have $G_{\delta}^{\prime}(s) \geq K G_{\delta}(s)$, and by integrating this over $(0, t)$, we arrive at

$$
\int_{X} \varphi\left(\sqrt{\left|D T_{t} f\right|^{2}+\delta^{2}}-\delta\right) d \mu \leq e^{-K t} \int_{X} T_{t} \varphi\left(\sqrt{|D f|^{2}+\delta^{2}}-\delta\right) d \mu .
$$

Passing to the limit $\delta \rightarrow 0$ and using the fact that the semigroup is self-adjoint, we finally obtain the desired inequality.

\section{REFERENCES}

[A] L. Ambrosio. Fine properties of sets of finite perimeter in doubling metric measure spaces, Set-Valued Anal. 10 (2002), 111-128.

[AD] L. Ambrosio and S. Di Marino. Equivalent definitions of BV space and of total variation on metric measure spaces, J. Funct. Anal. 266 (2014), 4150-4188.

[AGS] L. Ambrosio, N. Gigli, and G. Savaré. Bakry-Émery curvature-dimension condition and Riemannian Ricci curvature bounds, Ann. Probab. 43 (2015), 339-404.

[AMP] L. Ambrosio, M. Miranda jr., and D. Pallara. Special functions of bounded variation in doubling metric measure spaces, Calculus of variations: topics from the mathematical heritage of E. De Giorgi, Quad. Mat., Dept. Math., Seconda Univ. Napoli, Caserta, 14 (2004), 1-45.

[AMPP] L. Angiuli, M. Miranda jr., D. Pallara, and F. Paronetto. BV functions and parabolic initial boundary value problems on domains, Ann. Mat. Pura Appl. (4) 188 (2009), 297-331.

[Bakry] D. Bakry. Transformations de Riesz pour les semi-groupes symetriques. II. Étude sous la condition $\Gamma_{2} \geq 0$, Sé minaire de probabilité s, XIX, 1983/84, Lecture Notes in Math., 1123, Springer, Berlin, 1985, , 145-174.

[Ba] D. Bakry. L'hypercontractivité et son utilisation en théorie des semigroupes, in Lectures on probability theory (Saint-Flour, 1992), Lecture Notes in Math., 1581, Springer, Berlin, 1994, $1-114$.

[BE] D. Bakry and M. Émery. Diffusions hypercontractives, in Séminaire de probabilités, XIX, 1983/84, Lecture Notes in Math., 1123, Springer, Berlin, 1985, 177-206.

[BH] S. G. Bobkov and C. Houdré. Some connections between isoperimetric and Sobolev-type inequalities, Mem. Amer. Math. Soc. 129 (1997), viii+111.

[BMP] M. Bramanti, M. Miranda Jr., and D. Pallara. Two characterization of BV functions on Carnot groups via the heat semigroup, Int. Math. Res. Not. IMRN 17 (2012), 3845-3876.

[B] H. Brezis. How to recognize constant functions. A connection with Sobolev spaces, Uspekhi Mat. Nauk 57 (2002), 59-74.

$[\mathrm{CM}]$ A. Carbonaro and G. Mauceri. A note on bounded variation and heat semigroup on Riemannian manifolds, Bull. Austral. Math. Soc. 76 (2007), 155-160.

[C] J. Cheeger. Differentiability of Lipschitz functions on metric measure spaces, Geom. Funct. Anal. 9 (1999), 428-517.

[D] J. Dávila. On an open question about functions of bounded variation, Calc. Var. Partial Differential Equations 15 (2002), 519-527. 
[DeG] E. De Giorgi. Selected papers, Edited by Luigi Ambrosio, Gianni Dal Maso, Marco Forti, Mario Miranda and Sergio Spagnolo. Springer-Verlag, Berlin, 2006.

[DS] J.-D. Deuschel and D. W. Stroock. Large Deviations, Pure and Applied Mathematics, Academic Press, Boston, MA, 137, 1989 .

[FHK] B. Franchi, P. Hajłasz, and P. Koskela. Definitions of Sobolev classes on metric spaces, Ann. Inst. Fourier (Grenoble) 49 (1999), 1903-1924.

[FOT] M. Fukushima, Y. Oshima, and M. Takeda. Dirichlet forms and symmetric Markov processes, de Gruyter studies in mathematics, Walter de Gruyter and Co., Berlin 19, 1994.

[G] A. Grigor'yan. Analytic and geometric background of recurrence and non-explosion of the Brownian motion on Riemannian manifolds, Bull. Amer. Math. Soc. (N.S.) 36 (1999), 135249.

[GP] B. Güneysu and D. Pallara. Functions with bounded variation on a class of Riemannian manifolds with Ricci curvature unbounded from below, Preprint 2013, 1-26.

[HKT] T. Heikkinen, P. Koskela, and H. Tuominen. Sobolev-type spaces from generalized Poincaré inequalities, Studia Math. 181 (2007), 1-16.

[HK] J. Heinonen and P. Koskela. Quasiconformal maps in metric spaces with controlled geometry, Acta Math. 181 (1998), 1-61.

[HKST] J. Heinonen, P. Koskela, N. Shanmugalingam, and J. Tyson. Sobolev Spaces on Metric Measure Spaces: An Approach Based on Upper Gradients, New Mathematical Monographs, Cambridge University Press, Cambridge, UK 27, 2015.

[JK] R. Jiang and P. Koskela. Isoperimetric inequality from the Poisson equation via curvature, Comm. Pure Appl. Math. 65 (2012), 1145-1168.

[KKST] J. Kinnunen, R. Korte, N. Shanmugalingam and H. Tuominen. A characterization of Newtonian functions with zero boundary values, Calc. Var. Partial Differential Equations 43 (2012), $507-528$.

[KS] N.J. Korevaar and R.M. Schoen. Sobolev spaces and harmonic maps for metric space targets, Comm. Anal. Geom. 1 (1993), 561-659.

[KM] P. Koskela and P. MacManus. Quasiconformal mappings and Sobolev spaces, Studia Math. 131 (1998), 1-17.

[KRS] P. Koskela, K. Rajala, and N. Shanmugalingam. Lipschitz continuity of Cheeger-harmonic functions in metric measure spaces, J. Funct. Anal. 202 (2003), 147-173.

[L] M. Ledoux. Semigroup proofs of the isoperimetric inequality in Euclidean and Gauss space, Bull. Sci. Math. 118 (1994), 485-510.

[MMS] N. Marola, M. Miranda jr., and N. Shanmugalingam. Boundary measures, generalized GaussGreen formulas, and mean value property in metric measure spaces, Rev. Mat. Iberoam. 2 (2015), 1-34.

[M] V. Mazýa. Conductor and capacitary inequalities for functions on topological spaces and their applications to Sobolev-type imbeddings, J. Funct. Anal. 224 (2005), 408-430.

[Mr] M. Miranda jr. Functions of bounded variation on "good" metric spaces, J. Math. Pures Appl. 82 (2003), 975-1004.

[MP] M. Miranda Jr., D. Pallara, F. Paronetto, and M. Preunkert. Heat semigroup and functions of bounded variation on Riemannian manifolds, J. Reine Angew. Math. 613 (2007), 99-119.

[MP2] M. Miranda Jr., D. Pallara, F. Paronetto, and M. Preunkert. Short-time heat flow and functions of bounded variation in $\mathbb{R}^{N}$, Ann. Fac. Sci. Toulouse Math. (6) 16 (2007), 125-145.

[P-P] K. Pietruska-Pałuba. Heat kernels on metric spaces and a characterisation of constant functions, Manuscripta Math. 115 (2004), 389-399.

[P] M. Preunkert. A semigroup version of the isoperimetric inequality, Semigroup Forum 68 (2004), 233-245.

[S] G. Savaré. Self-improvement of the Bakry-Émery condition and Wasserstein contraction of the heat flow in $\operatorname{RCD}(K, \infty)$ metric measure spaces, Discrete Contin. Dyn. Syst. 34 (2014), $1641-1661$. 
[Sh] N. Shanmugalingam. Newtonian spaces: an extension of Sobolev spaces to metric measure spaces, Rev. Mat. Iberoamericana 16 (2000), 243-279.

[St1] K.-T. Sturm. Analysis on local Dirichlet spaces. I. Recurrence, conservativeness and $L^{p}$ Liouville properties, J. Reine Angew. Math. 456 (1994), 173-196.

[St2] K.-T. Sturm. Analysis on local Dirichlet spaces. II. Upper Gaussian estimates for the fundamental solutions of parabolic equations, Osaka J. Math. 32 (1995), 275-312.

[St3] K.-T. Sturm. Analysis on local Dirichlet spaces. III. The parabolic Harnack inequality, J. Math. Pures Appl. (9) 75 (1996), 273-297.

[W] F.Y. Wang. Equivalent semigroup properties for the curvature-dimension condition, Bull.Sci.Math. 135 (2011), 803-815.

(N.M.) Department of Mathematics and Statistics, University of Helsinki, P.O. Box 68 (Gustaf Hällströmin katu 2B), Fi-00014 University of Helsinki, Finland

E-mail address: niko.marola@helsinki.fi

(M.M) Department of Mathematics and Computer Science, University of Ferrara, via MachiaVelli 35, 44121, Ferrara, Italy

E-mail address: michele.miranda@unife.it

(N.S.) Department of Mathematical Sciences, University of Cincinnati, P.O.Box 210025, Cincinnati, OH 45221-0025, USA

E-mail address: shanmun@uc.edu 